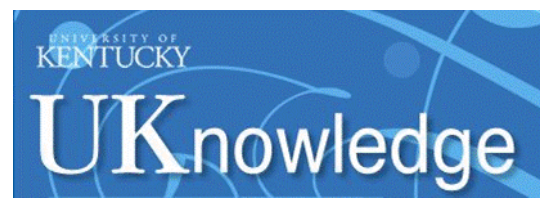

University of Kentucky

UKnowledge

2016

\title{
VOCALIZATION SUBSYSTEM RESPONSES TO A TEMPORARILY INDUCED UNILATERAL VOCAL FOLD PARALYSIS
}

\section{Daniel J. Croake}

University of Kentucky, djcroa2@uky.edu

Digital Object Identifier: https://doi.org/10.13023/ETD.2017.007

Right click to open a feedback form in a new tab to let us know how this document benefits you.

\section{Recommended Citation}

Croake, Daniel J., "VOCALIZATION SUBSYSTEM RESPONSES TO A TEMPORARILY INDUCED UNILATERAL VOCAL FOLD PARALYSIS" (2016). Theses and Dissertations--Rehabilitation Sciences. 36.

https://uknowledge.uky.edu/rehabsci_etds/36

This Doctoral Dissertation is brought to you for free and open access by the College of Health Sciences at UKnowledge. It has been accepted for inclusion in Theses and Dissertations--Rehabilitation Sciences by an authorized administrator of UKnowledge. For more information, please contact UKnowledge@lsv.uky.edu. 


\section{STUDENT AGREEMENT:}

I represent that my thesis or dissertation and abstract are my original work. Proper attribution has been given to all outside sources. I understand that I am solely responsible for obtaining any needed copyright permissions. I have obtained needed written permission statement(s) from the owner(s) of each third-party copyrighted matter to be included in my work, allowing electronic distribution (if such use is not permitted by the fair use doctrine) which will be submitted to UKnowledge as Additional File.

I hereby grant to The University of Kentucky and its agents the irrevocable, non-exclusive, and royalty-free license to archive and make accessible my work in whole or in part in all forms of media, now or hereafter known. I agree that the document mentioned above may be made available immediately for worldwide access unless an embargo applies.

I retain all other ownership rights to the copyright of my work. I also retain the right to use in future works (such as articles or books) all or part of my work. I understand that I am free to register the copyright to my work.

\section{REVIEW, APPROVAL AND ACCEPTANCE}

The document mentioned above has been reviewed and accepted by the student's advisor, on behalf of the advisory committee, and by the Director of Graduate Studies (DGS), on behalf of the program; we verify that this is the final, approved version of the student's thesis including all changes required by the advisory committee. The undersigned agree to abide by the statements above.

Daniel J. Croake, Student

Dr. Joseph Stemple, Major Professor

Dr. Esther Dupont-Versteegden, Director of Graduate Studies 


\section{VOCALIZATION SUBSYSTEM RESPONSES TO A TEMPORARILY INDUCED UNILATERAL VOCAL FOLD PARALYSIS}

DISSERTATION

A dissertation submitted in partial fulfillment of the requirements for the degree of Doctor of Philosophy in the College of Health Sciences at the University of Kentucky

By

Daniel J. Croake

Lexington, Kentucky

Director: Joseph C. Stemple, Professor of Communication Sciences and Disorders Lexington, Kentucky

2016

Copyright @ Daniel J. Croake 2016 


\section{ABSTRACT OF DISSERTATION}

\section{VOCALIZATION SUBSYSTEM RESPONSES TO A TEMPORARILY INDUCED UNILATERAL VOCAL FOLD PARALYSIS}

Healthy voicing is thought to be dependent on a dynamic balance of three interactive subsystems: respiration, phonation, and resonance. Theoretically, multiple patterns of subsystem interactions likely underlie healthy voice production; however surprisingly little quantitative data exists defining the nature of these subsystem production patterns and interactions across individuals.

The central aim of this study was to quantify the interactions of the vocalization subsystems in a non-perturbed and perturbed condition (induced unilateral vocal fold paralysis) in 10 vocally healthy participants. Respiratory inductance plethysmography, laryngeal aerodynamics, and acoustic formant data were used to measure the proportional contributions of, and changes to, the three vocal subsystems during voice production tasks. The overall hypothesis was that individuals would demonstrate distinctive patterns of change in voice subsystem interaction across vocalization conditions, resulting in characteristic vocalization profiles.

Using Dynamics Systems Theory (DST) as a guide, we hypothesized that analysis of group data alone would hide important individual variability that would help better understand differences in subsystem regulation across individuals. Additionally, in accordance with DST, we hypothesized that although there would be individual variability during voice production tasks, only a small group of characteristic subsystem patterns would emerge, permitting subgrouping of individuals into unique vocalization profiles.

Results demonstrated that group data masked important aspects of individual performance. Despite all individuals demonstrating paramedian paralysis on visualization during the perturbation phase, unique subsystem patterning strategies for coping with the acute vocal fold paralysis were observed. Despite individual variability, 
subgroups were able to be determined which revealed commonalities in the dominant physiologic strategies of subsystem regulation across individuals. A dynamic systems state space model was constructed as a visual aid to demonstrate that the changes noted between voicing conditions were not random, but rather formed specific trajectories. Implications for translation of these results into clinical practice are discussed.

KEYWORDS: Laryngeal, Voice, Respiratory, Aerodynamic, Resonance, Subsystem 


\title{
VOCALIZATION SUBSYSTEM RESPONSES TO A TEMPORARILY
} INDUCED UNILATERAL VOCAL FOLD PARALYSIS

\author{
By
}

Daniel J. Croake

Joseph C. Stemple

Chair

Esther Dupont-Versteegden

Director of Graduate Studies

$\underline{11-09-2016}$ 


\section{CHAPTER 1: INTRODUCTION}

Background.....

CHAPTER 2: REVIEW OF LITERATURE

Simple Versus Complex Systems......

Voice as a Complex System and Dynamic Systems Theory ..................... 10

Respiratory Kinematics....................................................... 12

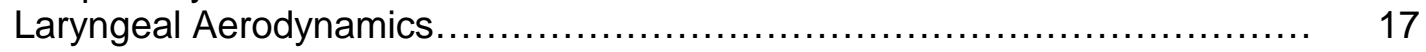

Acoustic Measures........................................................... 19

Relationships Among the Vocal Subsystems................................. 21

CHAPTER 3: METHODS

Research Design and Methods.............................................. 26

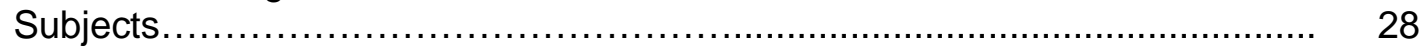

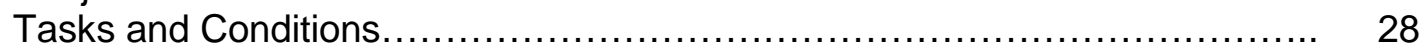

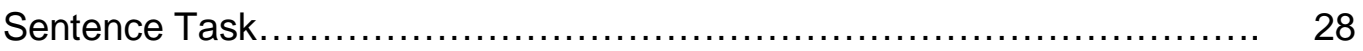

Measurement and Perturbation Techniques................................ $\quad 30$

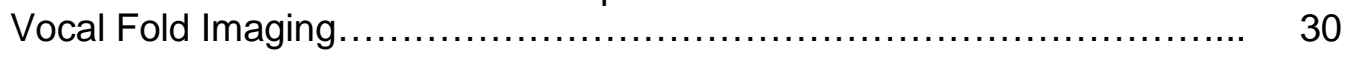

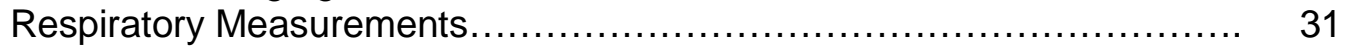

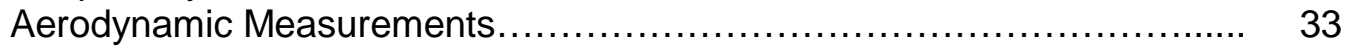

Acoustic Measurements................................................... 36

Laryngeal Perturbation Method......................................... 37

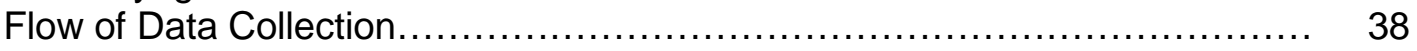

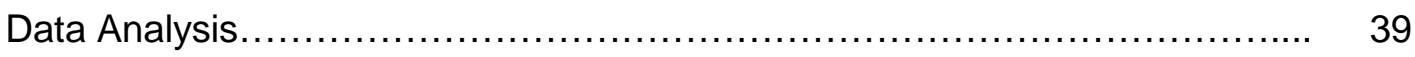

CHAPTER 4: RESULTS

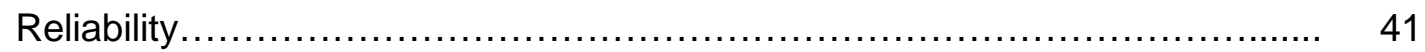

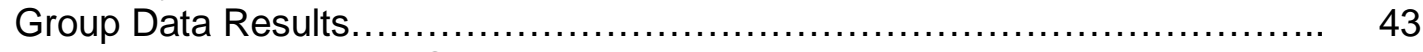

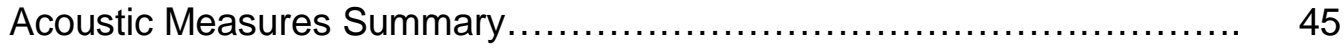

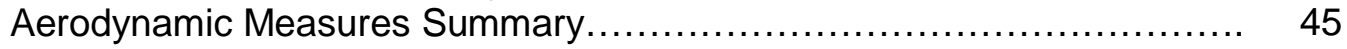

Respiratory Measures Summary ............................................ 46

CHAPTER 5: DISCUSSION

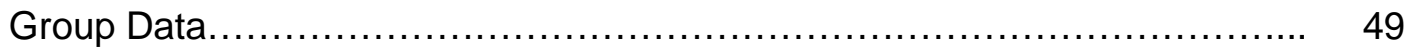

Acoustic Measures - Resonance Characteristics........................ 49

Aerodynamic Measures............................................. 50

Estimated Subglottic Pressure .................................... 51

Airflow Rate.......................................................... 51

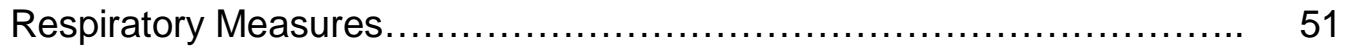

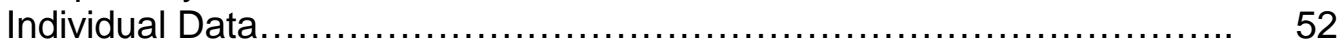

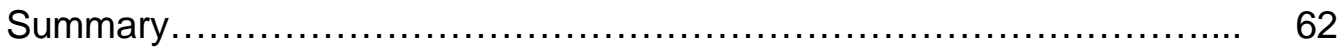

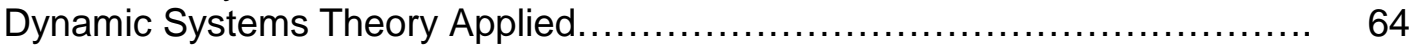

Lessons in Vocal Control in Relation to Dynamic Systems...................... 68

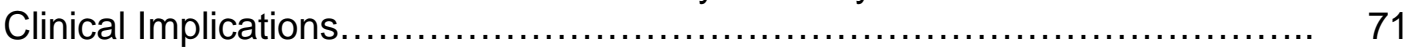

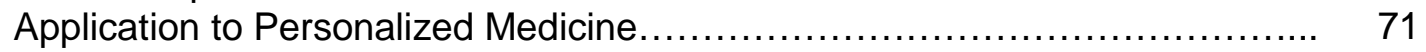

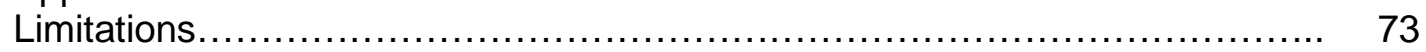

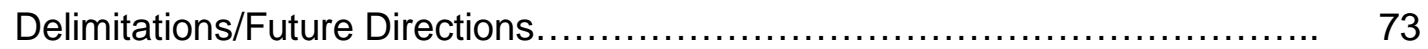




\section{LIST OF TABLES}

Table 2.1, Comparison of Normative Volumetric Data Across Studies.......... 15

Table 2.2, Comparison of Normative Aerodynamic Data Across Studies........ 19

Table 2.3, Comparison of Normative Acoustic Data Across Studies............. 21

Table 3.1, Definitions of Respiratory Measures................................ 27

Table 4.1, Demographic Subject Data......................................... 40

Table 4.2, Intraclass Correlations for Measurement Reliability.................. 41

Table 4.3, Descriptive Statistics, Means and Standard Deviations............... 43

Table 4.4, Repeated Measures ANOVA by Condition......................... 44

Table 5.1, Terms Relating to Discussion of Dynamic Systems Theory........... 64

\section{LIST OF FIGURES}

Figure 1.1, Traditional Model of Phonation................................... 7

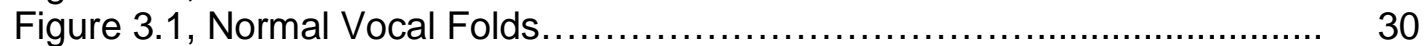

Figure 3.2 , Vocal Fold Paralysis .......................................... 31

Figure 3.3, Respiratory Kinematics: Lung Volume Initiations, Terminations, \& Excursions ........................................................ 33

Figure 3.4, Phonatory Aerodynamic System and Respiratory Inductance Plethysmography Setup......................................... 35

Figure 3.5, Airflow and Subglottic Pressure Example Traces................... 36

Figure 3.6, Spectrogram Example of Formant Values....................... 37

Figure 5.1, Percent Change by Subject for Formant Spacing between PRE \& PAR Conditions .................................................. 57

Figure 5.2, Percent Change by Subject for Aerodynamic Measures from PRE to PAR (Pressure, Flow) .......................................... 58

Figure 5.3, Percent Change by Subject from PRE to PAR for Lung Volumes... 58

Figure 5.4, Percent Change by Subject from PRE to PAR for Rib Cage Kinematics.

Figure 5.5, Percent Change by Subject from PRE to PAR for Abdominal

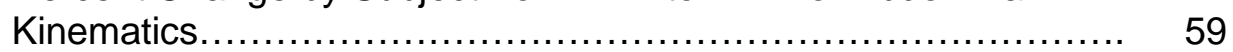

Figure $5.6+$ Respiratory Kinematic Plots by Subject......................... 60

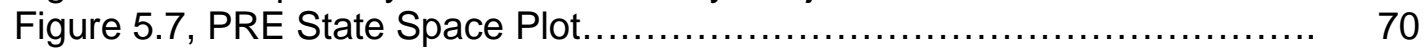

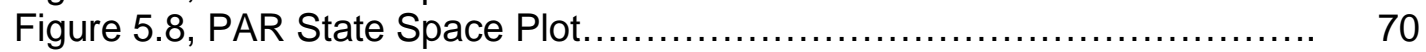

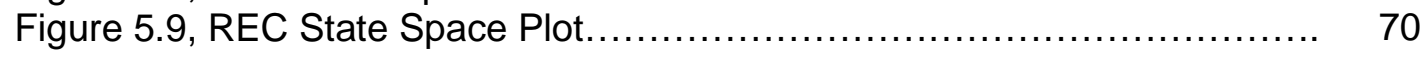

\section{APPENDICES}

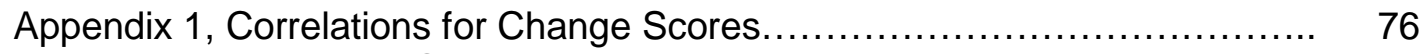

Appendix 2, Results by Subject............................................. 80

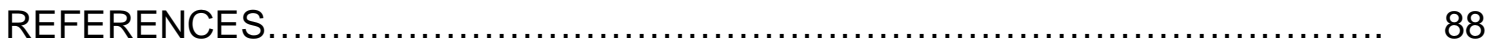

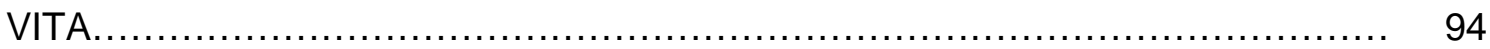




\section{CHAPTER 1: INTRODUCTION}

\section{Background}

Medical clinicians have long recognized that individuals with similar diagnoses may respond in dramatically different ways to the same course of treatment. It is clear that drugs targeted to treat a specific medical problem may result in a positive treatment response, no observable response, or even an adverse response. ${ }^{1-4}$ Personalized, and more recently, precision medicine seek to target therapy to a specific individual, recognizing that individual differences in body chemistry and genetic makeup may help to explain and predict treatment response. ${ }^{5}$

Similarly, the idea that there are important individual differences in phonatory regulation is not new. It is widely held that voice production is dependent upon a relative balance of the subsystems of respiration, phonation, and resonance; ${ }^{6-8}$ however there is a lack of empirical data that characterize how individuals regulate the vocal subsystems during both normal and disordered voice production. It is important to not only begin to acknowledge that individual differences in phonatory regulation exist, but also to characterize these differences in a quantitative way that permits clinical decision making. In order to move toward personalized voice treatment, it will be necessary to look beyond group averaging which masks individual variability and begin to routinely view variability as more than inconvenient noise to be eliminated. To develop more precision in voice treatment, a shift in thinking about study design and analysis with a greater focus on external validity will be required. The caveat to this paradigm shift is that it requires previous knowledge of measurements and techniques which have been demonstrated to be valid and reliable.

Fortunately, much is already understood about some important aspects of normal voice production. Normative data for the three vocalization subsystems has been 
established and are routinely used clinically to assess voice subsystem parameters; ${ }^{9-12}$ however there is another often underappreciated and overlooked problem in understanding regulation of phonatory behavior. This problem is the concept that voice production is complex and not simply a summation of each component subsystem. Rather, voice is an emergent behavior resulting from the complex interplay of variables both within and among the subsystem triad. Unfortunately, despite well described normative data for individual subsystems, much less is known about the interactive nature of the entire vocal system and the characteristic differences in individual regulation resulting in similar vocal output across individuals.

Additionally, the current model of phonation is typically viewed as a triad of vertically stacked and separated boxes which implies an evenly distributed bottom-up trajectory ${ }^{7,8}$. See Figure 1.1. This model is not well suited to describe subsystem interactions as it does not demonstrate that the subsystems do not function independently, but rather as a cohesive unit that is dynamically changing in the context of phonation across individuals The current model also does not acknowledge the multiple combinations of variables within each subsystem that contribute to the overall contour of the entire system. Our current conceptual model should be updated in a way that better describes the realities that occur both within and across individuals during voicing. The current conceptual model of voice production warrants a revision in order to display a more detailed, yet simple view of voice production. To this end, the complexities by which vocal output is regulated across individuals and the proportional contributions of the subsystem triad should be measured simultaneously in a way that reveals how the most dominant variables within each subsystem shape and influence the dynamics of the system as a whole. 
This research project aimed to describe and quantify the proportional relationships among the vocalization subsystem triad in relation to both group and individual performance during voice tasks in a normal state, a perturbed state, and a post-perturbation recovery state. Dynamic systems theory was used as a theoretical framework and rationale to support the idea that voice is a complex system and therefore warrants not only a reductive analysis, but also holistic analyses to provide a richer understanding of the data. ${ }^{13}$

The research design of this project was based on "practical clinical trials" which seek to focus more strongly on external validity. ${ }^{14,15}$ Studies of this nature loosen internal controls to permit observation of performance in more natural conditions. Because one goal of this study was to observe similarities and differences in individual voice production, vocal tasks were not controlled with regard to frequency and intensity. It is known that reliability of vocal tasks increases when these variables are controlled, ${ }^{16}$ however it was of greater interest to observe how individuals perform vocal tasks when left to their own devices. This may be viewed as a weakness in experimental design; however much is already known about the reliability and validity of the measures and variables used in this study. Because there is a foundation for the measurement techniques and established normative data there is a strong basis from which inferences can be made regarding these measures. Additionally, individual differences would be masked with tight controls in place during voice tasks. Because it is the individual differences and similarities that are of interest in this study the choice was made to loosen internal controls as controlled data has been reported previously and is reviewed in chapter two.

To expand on the small body of work concerning the interactive nature of the vocalization subsystems, the objective of this study was to further investigate the 
proportional contributions of the respiratory, phonatory, and resonance systems before, during, and after a fictive and reversible perturbation of temporarily induced unilateral vocal fold paralysis (UVFP) in 10 adults without voice problems. Vocal fold paralysis is a common voice pathology which impairs the valving action of the vocal folds resulting in decreased airway resistance at the level of the glottis due to air leak which interrupts the pressure and flow dynamics of the system. Disturbance of the normal patterns of pressure and flow through the vocal folds alters the internal balance of the vocalization subsystems and evokes a response from the voice user to compensate for the disruption of pressure and flow. Voice pathologies in general, and their associated changes in voice quality are thought to result from a disturbance to one or more of the vocalization subsystems resulting in an imbalance in overall system performance. ${ }^{8}$ Temporary vocal fold paralysis was chosen as the perturbation method for this study because it 1) permits disturbance to the laryngeal complex without mechanically altering the oral cavity, which permits measurement of formant activity, reflecting changes in vocal tract posture, and 2) is a frequently encountered clinical voice pathology which is not often able to be assessed in the acute phase. Therefore it is not well known how individuals respond to this pathological state acutely.

The overarching hypothesis for this study was that individuals would demonstrate characteristic and distinctive patterns of vocal subsystem adaptations, resulting in identifiable vocalization profiles. Simultaneous measures of respiratory kinematics, laryngeal aerodynamics, and vocal tract acoustic formant spacing were taken representing the three subsystems of phonation. The following specific aim was constructed to address the following experimental question: Does a perturbation to the laryngeal complex (induced UVFP) result in identifiable and distinct compensatory 
changes in the subsystem patterning within and across individuals, or are compensatory behaviors entirely idiosyncratic?

Specific Aim: Quantify the interactive tradeoffs among respiration, phonation, and resonance in individuals with induced UVFP during the production of vocalization tasks.

Using repeated measures, we tested the hypothesis that an induced physiologic perturbation to the laryngeal complex would result in the adoption of identifiable and distinct compensatory changes in subsystem patterning compared to control.

An important outcome of this aim was to operationally characterize the nature of subsystem interactions in the normal, perturbed, and acute recovery phase. We hypothesized that multiple combinations of subsystem interactions would result revealing individual characteristic patterns of compensation, and also commonalities within the cohort (i.e. predominantly laryngeal driven, or predominantly respiratory driven voicing).

This study was developed to extend the results of previous work and provide novel information regarding the compensatory strategies of individuals during a short-term pathological condition. The overall goal of this study was to 1) determine if a characteristic normal vocalization subsystem pattern emerged during non-perturbed vocalization tasks when subjects were permitted to use their typical vocalization habits, and 2) extend these findings via a perturbation design that permitted measurement of respiration, phonation, and resonance in the context of a common vocal pathology. For the non-perturbed phase of this study, specific hypotheses for respiratory, laryngeal, and resonance were made based on previous literature and are as follows: 
- There would be more than one characteristic patterning of the vocalization subsystems indicating individual preference for a more respiratory, or laryngeal driven strategy for phonation.

- The individual subsystem measurements would generally be in agreement with published normative data.

During the perturbation phase of the study, it was hypothesized that:

- There would be a general increase in respiratory initiations, terminations, and excursions; and increased subglottic pressure and average airflow rate.

- Distance between normalized formant values (measured in "cents") would be altered indicating supraglottic (resonance) alterations to compensate for the predominantly open phase of vocal fold closure during temporary paralysis.

We also hypothesized that interactive patterns would be variable from subject to subject; however we expected only a small group of characteristic patterns to emerge.

In order to provide context for this study, chapter two will review the concepts of simple and complex systems and introduce the framework of dynamic systems theory in relation to voice production. Previous relevant literature regarding the three subsystems of phonation will be reviewed along with the small body of literature that has discussed the interactive nature of the subsystem triad. 


\section{Figure 1.1}

Traditional Model of Phonation. The three boxes represent a "bottom-up" representation of vocalization where respiration is the pulmonary driving force, phonation is the level of the vocal folds which are set into vibration due to respiratory airflow, and resonance which represents the acoustic tube above the larynx which filters the acoustic energy from the vibration of the vocal folds.

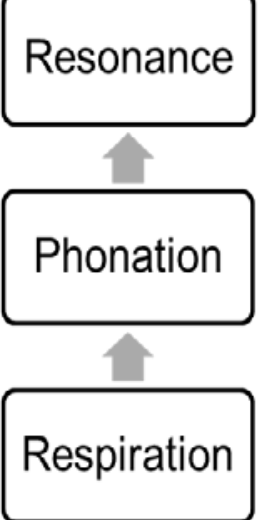




\section{CHAPTER 2: REVIEW OF LITERATURE}

This chapter provides a brief introduction to simple and complex systems. Next, relevant literature pertaining to the three vocalization subsystems is reviewed. Finally, voice production is discussed using the framework of dynamic systems theory to provide context for our experimental design.

\section{Simple versus Complex Systems}

Simple systems function in a predictable (linear) and additive manner where the observed output of the system is always determined by the additive contributions of its components. They produce specific and repeatable patterns of output or behavior that can be reliably predicted. Simple systems are, in general, more easily studied because of their linear and additive properties as the workings of a simple system can always be theoretically dissected and re-summed until the function of each component part is understood.

Additionally, simple systems are not influenced by internal or external environmental factors, therefore the behavior of the system is always the same regardless of context. Briefly, a system may be considered simple if it: 1) contains few (or many) component parts or elements that have a predictable function, 2) has few interactions among its parts, 3) does not evolve over time, and 4) is not affected by the environment or behavior. Clocks, despite their numerous moving parts are simple systems because their behavior is linear and predictable. Each piece of the inner workings has a known and predictable function which when added together always results in the same predictable outcome.

In contrast to simple systems, complex (dynamic) systems consist of interacting components whose output cannot be predicted by simple addition. Complex systems 
may be linear (periodic but complex), but are often non-linear (aperiodic). The components in a complex dynamic system may execute a specific, well known function independently; however the components may function differently in the context of the whole system due to influences that come about through interactions, context, and chance. $^{13}$

The interactive nature of complex systems influences system output into particular organizational patterns within a particular context. The interactions can potentially lead to a variety of possible outcomes, however complex systems typically exhibit "preference" to behave in a particular manner. This preferential convergence is highly context dependent. This means that the predictability of the system is unknown unless it is directly observed because internal and external factors have influence over the functional nature of the system. These additional properties make the study of complex systems more challenging. We view voice as a complex and non-linear system because vocal output is not directly proportional to its input. Pressure from the lungs is modulated differently in individuals as it is transduced to airflow and pumped through the vocal tract. Additionally, vocal output is context dependent and is continually changing during speech due to prosodic influences. In other words, vocal output is not necessarily predictable as the component subsystems may function very differently to produce vocal output both within and across individuals.

The non-linearity and non-additivity of complex systems poses a problem in making sense of a system's function if it is viewed in the same manner as a simple system. A different approach requiring observation of the entire system in a particular context is necessary because, regardless of fundamental knowledge of the component parts, they cannot be simply added together as doing so offers a distorted view of the overall system function. 


\section{Voice as a Complex System and Dynamic Systems Theory}

We have previously stated that vocal output is an emergent behavior resulting from complex dynamic interactions. It is therefore important to appreciate that vocal function should not be viewed as simply the sum of the component subsystems. Instead, observation of the simultaneous interactive nature of the subsystem triad is appropriate to better appreciate how the vocal subsystems collect themselves in different contexts.

This gestalt approach is derived from Chaos Theory, and is known as Dynamic Systems Theory (DST). Use of such an approach has proven useful in other scientific fields such as Systems Biology because of the inherent limitations of the traditional reductionist approach. ${ }^{17-20}$ Where a reductionist approach would seek to continually reduce complex phenomena into its simplest component features, DST recognizes that it is not simply the understanding of the smallest elements, but rather how those elements function in a given context to yield additional valuable information regarding the gross function of a complex system, i.e., the elements of a dynamic system cannot simply be added together to reveal the determinants of system behavior. ${ }^{13}$

Instead, DST postulates that because the component parts of complex systems cannot reliably predict system outputs, (the system is not additive and often non-linear) it is therefore not advantageous to dissect a system's individual components, but rather to study the emergent organizational patterns brought about by component interactions in a given condition and context. ${ }^{13}$ Study of the interactive nature of a system may be accomplished by perturbing a system (disturbing normal functional state in a given context) and observing how the system compensates. The observed transitions often reveal details in the overall function of the system. A transition occurs when one preferred state of behavior is replaced by another new form of behavior. ${ }^{13}$ 
As stated previously, complex systems have preferred organizational patterns which converge from the cooperative interplay of components. DST terms these preferred patterns as "attractor states." Attractor states are stable or efficient functional modes of a system in a given condition. There are often a number of context dependent possibilities in which a system could collect itself; however attractor states tend to "suck in" the system into a particular convergence patterns. Attractor states cannot be predicted by decomposition of a system's component parts. Rather, only gestalt observation can reveal the determinants which converge into overall system behavior.

Attractors have been likened to "basins" into which particular behavioral pattern "settle in." The basin occupies a particular space (state space) in which a behavioral pattern remains loosely stable; however if the system is given a "push" with enough force, a behavior can be disrupted from its attractor basin. When this occurs, the system is thrown into instability, breaking convergence and "seeks out" a new context in which to become stable again. If a state space is available in the new context, the system will attempt to converge in a new manner to stabilize the system behavior. Perturbing a system is a useful way to study system dynamics because transitional states can reveal the boundaries of the normal function of the system. Moreover, observation of how the system collects itself can reveal vital information regarding the governance of the system.

Holistic approaches like DST, allow for "big picture" thinking about the way systems behave and therefore can provide information about system function that the narrowly focused lens of reductionism does not permit. However, the tradeoff is that holistic approaches can only supply information regarding overall system performance because the individual components of the system are not predictable and thus cannot be known in detail. 
The DST approach does not seek to reduce variability in search of a single target solution, but rather views phenomena as having a cluster, or cloud-like arrangement of normal function. Variability is not viewed as noise to be eliminated, but rather a rich source of information that is both useful and necessary to describe the function of a system. A marked increase in variability of a system can indicate that a system shift may be eminent. Thus perturbation of the system can be exploited in order to map boundaries of interesting behaviors.

We believe that Dynamic Systems Theory is a useful heuristic to study voice production because it permits study of the non-linear complexities of biological systems.

What is known about collective vocal function has been largely inferred from individual studies of the subsystems of voice production. Only a few studies have used simultaneous measures to describe the dynamic nature of respiration, phonation, and resonance during normal and perturbed voice production revealing characteristic differences across conditions both within and across subjects. A brief review of the literature for each subsystem and those studies which have used simultaneous measures will be reviewed.

\section{Respiratory Kinematics}

Respiratory kinematic measurements permit qualitative and quantitative metrics of the relative motion of the chest wall (ribcage and abdomen) in relation to the total lung volume used during a speech task. The movements of the ribcage and abdomen may be calibrated into a volume index either by isovolume maneuvers, or by using a least squares calculation. ${ }^{11,21-23}$

Chest wall configurations have been classically studied using magnetometry, and more recently using respiratory inductance plethysmography. ${ }^{11,21,24-27}$ Both methods provide 
volume indices of the proportional contributions of the rib cage and abdomen during speech tasks with comparable results as evidenced by similar numeric data in the literature. It has been generally observed that the rib cage is the predominant contributor to the total lung volume changes during speech tasks in upright position, while the abdomen plays a larger role in supine position. ${ }^{11,21}$ Studies generally demonstrate that at comfortable loudness, speech is produced within the midrange of subjects' lung volumes, typically initiated above end expiratory level (EEL) and terminated at, or slightly above EEL. End expiratory level is the point of passive relaxation where lung pressure and atmospheric pressure differentials are matched and therefore no air is moving in or out of the lungs. This point typically occurs at $35-40 \%$ of vital capacity. ${ }^{28}$ In general, lung volumes used for speech may encompass anywhere from 20 - 70\% of an individual's vital capacity. The lung volume used to initiate speech production is about 50 $-60 \%$ of vital capacity, and the volume at which speech is terminated has been shown to be anywhere from $50-30 \%$ of vital capacity. ${ }^{11}$ The mean lung volume range during reading tasks has been reported by one study to be $39 \%{ }^{29}$

Other studies have reported lung volumes in terms of the volume used relative to EEL. (Also reported as resting expiratory level, or REL). Generally these studies report speech initiation volumes above EEL by 9 - 16\%. Lung volume terminations are typically at or below EEL and range from $0.5 \%$ above EEL to $-5 \%$ below EEL. ${ }^{30,31}$

Collectively, data indicate inherent variability in chest wall movement used to generate the pressures needed for healthy voicing. This is further evidenced by studies that report high intra and intersubject variability in patterning of the chest wall during speech breathing. ${ }^{27,30,32}$ Average measurements across studies are generally in agreement; ${ }^{25-27,30,31}$ however considerable variability has been reported when observing individual data indicating that averaging hides important components of individual 
variability. ${ }^{27}$ Unfortunately, direct data comparisons are not possible across many studies due to differences in methodology and data reporting. Table 2.1 provides a comparison across studies which report mean kinematic data for lung volumes, and ribcage and abdomen excursions. While in broad agreement, considerable variability can be appreciated. The high levels of variability are of interest not because they indicate measurement error, but rather because of the many degrees of freedom that can be achieved by the chest wall in order to maintain a constant subglottic pressure source for vocalization while altering these pressures for prosodic variety. 
TABLE 2.1

Mean volumetric data reported as \% of vital capacity and relative to EEL for healthy

Subjects during syllable trains and sentence tasks. Lung volume initiation,

termination, and excursion (LVI, LVT, LVE), rib cage initiation, termination,

and excursion ( $\mathrm{RCI}, \mathrm{RCT}, \mathrm{RCE})$ and abdominal initiation, termination, and excursion

$(A B I, A B T, A B E)$ are reported.

\begin{tabular}{|c|c|c|c|c|c|}
\hline Measure & Study & \multicolumn{2}{|c|}{ Men } & \multicolumn{2}{|c|}{ Women } \\
\hline & & Mean & SD & Mean & SD \\
\hline LVI & $\begin{array}{l}\text { a. } \\
\text { b. } \\
\text { c. } \\
\text { d. }\end{array}$ & $\begin{array}{c}13.69 \\
21.07 \\
\text { n/a } \\
21.03^{\star}\end{array}$ & $\begin{array}{c}4.52 \\
11.86 \\
\mathrm{n} / \mathrm{a} \\
\mathrm{x}\end{array}$ & $\begin{array}{c}21.01 \\
13.39 \\
15.08 \\
\star\end{array}$ & $\begin{array}{c}12.01 \\
4.38 \\
6.5 \\
x\end{array}$ \\
\hline LVT & $\begin{array}{l}\text { a. } \\
\text { b. } \\
\text { c. } \\
\text { d. }\end{array}$ & $\begin{array}{c}-6.87 \\
-4.30 \\
\mathrm{n} / \mathrm{a} \\
-9.82^{*}\end{array}$ & $\begin{array}{c}6.33 \\
8.55 \\
\mathrm{n} / \mathrm{a} \\
\mathrm{x}\end{array}$ & $\begin{array}{c}-4.59 \\
-6.53 \\
2.58 \\
\star\end{array}$ & $\begin{array}{c}8.62 \\
6.37 \\
8.5 \\
x \\
\end{array}$ \\
\hline LVE & $\begin{array}{l}\text { a. } \\
\text { b. } \\
\text { c. } \\
\text { d. }\end{array}$ & $\begin{array}{c}20.56 \\
25.81 \\
\mathrm{n} / \mathrm{a} \\
30.85^{\star}\end{array}$ & $\begin{array}{c}5.60 \\
7.03 \\
\mathrm{n} / \mathrm{a} \\
\mathrm{x}\end{array}$ & $\begin{array}{c}25.60 \\
19.93 \\
12.51 \\
*\end{array}$ & $\begin{array}{c}6.81 \\
5.54 \\
7.8 \\
x\end{array}$ \\
\hline $\mathrm{RCl}$ & $\begin{array}{l}\text { a. } \\
\text { b. } \\
\text { c. } \\
\text { d. }\end{array}$ & $\begin{array}{c}12.03 \\
27.23 \\
\text { n/a } \\
19.38^{*}\end{array}$ & $\begin{array}{c}4.16 \\
41.42 \\
\mathrm{n} / \mathrm{a} \\
\mathrm{x}\end{array}$ & $\begin{array}{c}18.47 \\
12.67 \\
14.51 \\
*\end{array}$ & $\begin{array}{c}15.36 \\
3.65 \\
7.6 \\
x\end{array}$ \\
\hline RCT & $\begin{array}{l}\text { a. } \\
\text { b. } \\
\text { c. } \\
\text { d. }\end{array}$ & $\begin{array}{c}-5.65 \\
-1.51 \\
\mathrm{n} / \mathrm{a} \\
-14.80^{*}\end{array}$ & $\begin{array}{c}6.85 \\
21.02 \\
\mathrm{n} / \mathrm{a} \\
\mathrm{x}\end{array}$ & $\begin{array}{c}-1.69 \\
-3.26 \\
-2.69 \\
*\end{array}$ & $\begin{array}{c}12.13 \\
4.16 \\
10.3 \\
x\end{array}$ \\
\hline RCE & $\begin{array}{l}\text { a. } \\
\text { b. } \\
\text { c. } \\
\text { d. }\end{array}$ & $\begin{array}{c}17.68 \\
25.76 \\
\text { n/a } \\
34.18^{\star}\end{array}$ & $\begin{array}{c}6.64 \\
23.26 \\
n / a \\
x\end{array}$ & $\begin{array}{c}20.23 \\
19.58 \\
17.19 \\
*\end{array}$ & $\begin{array}{c}7.99 \\
8.04 \\
7.6 \\
x\end{array}$ \\
\hline ABI & $\begin{array}{l}\text { a. } \\
\text { b. } \\
\text { c. } \\
\text { d. }\end{array}$ & $\begin{array}{c}11.34 \\
15.87 \\
\text { n/a } \\
10.58^{*}\end{array}$ & $\begin{array}{c}4.56 \\
9.21 \\
\mathrm{n} / \mathrm{a} \\
\mathrm{x}\end{array}$ & $\begin{array}{c}11.49 \\
12.59 \\
9.27 \\
*\end{array}$ & $\begin{array}{c}6.66 \\
2.99 \\
7.4 \\
x\end{array}$ \\
\hline ABT & $\begin{array}{l}\text { a. } \\
\text { b. } \\
\text { c. } \\
\text { d. }\end{array}$ & $\begin{array}{c}-7.64 \\
-9.42 \\
\text { n/a } \\
-16.91^{\star}\end{array}$ & $\begin{array}{c}5.83 \\
6.53 \\
\mathrm{n} / \mathrm{a} \\
\mathrm{x}\end{array}$ & $\begin{array}{c}-12.48 \\
-6.34 \\
-7.11 \\
*\end{array}$ & $\begin{array}{c}6.45 \\
6.78 \\
12.6 \\
x\end{array}$ \\
\hline ABE & $\begin{array}{l}\text { a. } \\
\text { b. } \\
\text { c. } \\
\text { d. }\end{array}$ & $\begin{array}{c}20.32 \\
25.28 \\
\text { n/a } \\
27.48^{\star}\end{array}$ & $\begin{array}{c}8.47 \\
10.43 \\
\mathrm{n} / \mathrm{a} \\
\mathrm{x}\end{array}$ & $\begin{array}{c}24.40 \\
18.96 \\
16.38 \\
*\end{array}$ & $\begin{array}{c}10.14 \\
7.33 \\
10.7 \\
x\end{array}$ \\
\hline
\end{tabular}

a.) Stathopolous and Sapienza, 1993; b.) Stathopolous and Sapienza, 1997; c.) Huber et al., 1998; d.)

*Huber and Stathopolous, 2003 (*study reported combined data for men and women as no significant sex differences were found for any lung volume measurements). 
Studies that have used syllable tasks rather than conversation or reading tasks have demonstrated greater consistency during respiratory measures, however there appears to be a wide range of variability regardless of the task used. It may be seen from Table 2.1, that large standard deviations are present for respiratory kinematics in both syllable trains and sentences. Winkworth systematically studied speech breathing during reading and spontaneous speech expanding on Hixon's work. These studies reported high levels of both within and between subject variability in lung volume measurements. ${ }^{26,27}$ The percent of total variation attributed to intersubject variance was $24.85 \%$ for lung volume initiations (LVI), and $25.64 \%$ for lung volume terminations (LVT). For intrasubject variance the percent of total variation was $46.48 \%$ for $\mathrm{LVI}$, and $46.06 \%$ for LVT. Greater variability would be expected during non-restricted speech tasks because of prosodic influences as well as fluctuating utterance length, and this was indeed the case in the Winkworth et al. studies.

Studies which have systematically altered speech tasks by increasing intensity level have generally observed that as loudness increases overall lung volumes increase, ribcage initiations are higher and abdominal volumes are lower indicating active muscular action of the abdominal musculature to provide structural support for increased lung pressures. ${ }^{33,34}$ One study has measured respiratory kinematic changes during a perturbation to oral cavity via an oral tube designed to create an oral pressure bleed during vocalization. With increasing loss of oral pressure, subjects inhaled to larger (but not significantly larger) lung volumes and generally responded similarly to subjects who are attempting to raise vocal intensity. ${ }^{35}$

Although respiratory kinematic studies have been in general agreement regarding some parameters, the individual data demonstrate that some subjects do not follow the general trends exemplified by group data. For example, Stathopolous and 
Sapienza reported three different responses in respiratory kinematics with cues to increase vocal intensity. ${ }^{33}$ These strategies involved differences in the combined use of the respiratory and laryngeal systems in order to increase vocal intensity. This finding reinforces the dynamic interactions present among the vocal subsystems, and also reveals that individuals have differing strategies in order to adapt to changing vocal intensity (a change in context).

\section{Laryngeal Aerodynamics}

Aerodynamic measures permit indirect measurement of the pressures and flows generated by the lungs and by the valving action of the larynx during vocalization. A recent normative database for the commercially available KayPENTAX Phonatory Aerodynamic System (PAS) has recently been reported. ${ }^{10}$ Regardless of the system used, aerodynamic measures require a transduction of oral pressure to be used as an estimate of subglottic pressure and oral airflow during vocal tasks. An anesthesia type mask coupled with a pneumotachometer is typically used to measure the oral airflow during voicing. Subglottic pressure is estimated orally by a small-diameter flexible tube placed in the mouth that senses pressure generated during an unvoiced pressure consonant, typically /p/. Because lung pressure is transmitted rapidly to the oral cavity when the lips and velum are closed, the oral estimate is a very close approximation of the lung pressure generated for voicing. ${ }^{36}$ A circumvented mask, Rothenberg type (1973) or a non-vented mask may be used, but the basic principle is the same with either type. Briefly, airflow is sensed by a drop in pressure across a known resistance and pressure is transduced via an oral tube. The circumvented mask permits inverse filtering of the oral airflow waveform. ${ }^{37}$ Although inverse filtering offers finer measurements and permits characteristics of individual glottal pulses, there is currently no commercial system that completely automates this process and therefore is not 
typically used clinically. More gross measurements of mean airflow rate, and transoral pressure are easily collected with a non-circumvented anesthesia-type mask with commercially available instrumentation and automated software programs. Although a concern, it has been shown that the type of mask used (vented or non-vented) does not significantly affect respiratory patterns during vocal tasks. ${ }^{38,39}$

Many measures can be made with aerodynamic instrumentation, however two commonly used and clinically useful measurements that describe laryngeal valving are: estimated mean subglottic pressure measured orally in $\mathrm{cm} \mathrm{H} 20\left(P_{s}\right)$ and mean airflow rate (flow) measured in $\mathrm{mL} / \mathrm{s}$. Table 2.2 reports normative data for these measures. Studies reported here have used both the KayPENTAX system and inverse filtering methods. It can be seen that both pressure and flow measures are in general agreement across studies; however the ranges can be quite large indicating that some subjects may be employing differing strategies within the confines of the normative data. All pressure and airflow data are in agreement with classic studies that report $P_{s}$ to be from 5-10 $\mathrm{cmH} 20$, and mean airflow rates to be $70-200 \mathrm{~mL} / \mathrm{s}$. Hirano reported that these values have large individual variability and that values above $400 \mathrm{~mL} / \mathrm{s}$ should be considered abnormal. ${ }^{6,40,41}$ A recent study of intrasubject test-retest reliability for the KayPENTAX PAS system using intraclass correlation coefficients reported and ICC of 0.81 for mean airflow rate, and 0.74 for estimated subglottic pressure. ${ }^{42}$ 


\section{Table 2.2}

Comparison of four studies reporting data for mean estimated subglottic pressure and oral airflow. Studies a, c, and d used repetitions of $/ p a /$, while study b used /pae/.

\begin{tabular}{|l|l|l|l|l|l|}
\hline Measure & Study & \multicolumn{2}{|l|}{ Men } & Women \\
\hline & & Mean (SD) & Range & Mean (SD) & Range \\
\hline Mean peak air & a. & $6.65(1.98)$ & $3.59-11.43$ & $5.40(1.37)$ & $2.52-8.68$ \\
pressure (cm & b. & $6.30(1.4)$ & $4.20-9.60$ & $5.80(0.9)$ & $4.40-7.60$ \\
& c. $)$ & $5.21(1.30)$ & $x$ & $4.75(1.22)$ & $x$ \\
& d. & $5.23(1.16)$ & $x$ & $4.07(1.11)$ & $x$ \\
\hline Mean airflow & a. & $0.14(0.08)$ & $0.01-0.37$ & $0.10(0.05)$ & $0.02-0.21$ \\
during & boicing (L/s) & $0.19(0.07)$ & $0.1-0.3$ & $0.14(0.03)$ & $0.09-0.21$ \\
& c. & $0.12(0.04)$ & $x$ & $0.12(0.03)$ & $x$ \\
& d. & $0.12(0.04)$ & $x$ & $0.11(0.03)$ & $x$ \\
\hline
\end{tabular}

a.) Zraick et al., 2012; (using KayPENTAX PAS System), b.) Holmberg, Hillman, and Perkell, 1988;

c.) Stathopopous and Sapienza, 1993; d.) Stathopolous and Sapienza, 1997.

\section{Acoustic Measures}

Acoustic measures provide indirect information about the resonance properties of the vocal tract. Changes in vocal tract shape act to filter the glottal source spectra which alters the acoustic output in predictable ways. ${ }^{43}$ A spectrogram displays the frequency, intensity, and temporal characteristics of an acoustic signal. Areas of enhanced resonance of the vocal tract called formants can be measured using spectrographic analysis. Formant frequencies and their relative spacing are useful means to characterize vocal output. Lower formants (F1, F2) are strongly correlated with the perception of vowels and are created primarily from lingual and oral positioning. Higher formants (F3, F4, F5) also reflect vowel space, but are additionally associated with the perception of characteristic vocal qualities. ${ }^{44}$ Vocal quality, or timbre has been shown to be altered in part by laryngeal elevation/depression and by the length and cross- 
sectional area of the pharynx above the vocal folds referred to as the epilarynx tube. ${ }^{45,46}$ Overall changes in the vocal tract shape, as well as changing vocal fold vibratory characteristics may function to change formant patterning during voicing. ${ }^{46,47}$ For example, increased vocal intensity is associated with increased fundamental frequency and oral cavity opening, both of which can alter formant intensity and spacing. ${ }^{48}$

Supraglottic compression is a common characteristic of voice disorders. The musculature above the vocal folds may narrow and squeeze in attempt to achieve better true vocal fold closure in the case of glottic insufficiency. Although typically considered maladaptive in speech production, some singing styles purposefully cultivate some degree of epilaryngeal narrowing (along with pharyngeal widening) in order to boost acoustic energy in the $2.5-3.5 \mathrm{kHz}$ range. Sundberg has called this phenomenon the "singer's formant cluster" and is primarily in the region of formants $3-5 .{ }^{45}$ During epilaryngeal narrowing the spacing of these formants has been shown to narrow or cluster together creating a characteristic "ringing" vocal quality that can carry over an orchestra without artificial amplification. Enhanced resonance allows for greater acoustic power with less effort and thus is more efficient and economical for the voice user. This economical type of voice production is the typical biomechanical target of voice therapy and can be facilitated through use of a semi-occluded vocal tract. ${ }^{49-51}$

Much less is known about upper formants with the exception of their role in the singing voice; however one recent case study determined the effect of straws and resonance tubes on the shape of the pharynx using CT scanning and acoustic formant measures. After using both a straw and a specially designed resonance tube, the subject demonstrated a significant narrowing in the spacing of formants F3-F5 with the largest narrowing occurring between F4 and F3. The greatest change in formant distance was over 35\% from baseline. This study demonstrated that a relative narrowing of the 
epilarynx tube (or expansion of the pharynx) alters the relative spacing of the upper formant frequencies. ${ }^{52}$

Not unlike previous measures, mean data for formant values of men and women have been established. Unfortunately, there are no studies which report coefficients of variability for these measures; however it is known that the linear predictive coding settings are the source of the greatest variance for these measures and therefore these settings were kept constant for analysis of these measures. ${ }^{53}$ The range of mean values for formants F1- F3 across four different studies are listed in Table $\mathbf{2 . 3}$ for reference.

\section{TABLE 2.3}

Mean frequency ranges across studies for $F_{0}-F 3$.

\begin{tabular}{|l|l|l|l|l|}
\hline & \multicolumn{2}{|l|}{ Men } & Women & /J/ \\
\hline Vowel & /a/ & /J/ & /a/ & $210-216$ \\
\hline$F_{\text {o }} \mathbf{H z}$ & $124-135$ & $121-129$ & $212-231$ & $590-781$ \\
\hline F1 Hz & $723-768$ & $570-653$ & $850-936$ & $920-1136$ \\
\hline F2 Hz & $1090-1333$ & $840-1048$ & $1220-1551$ & $2710-2986$ \\
\hline F3 Hz & $2440-2522$ & $2410-2599$ & $2810-2950$ & \\
\hline
\end{tabular}

Sources: Complied in Baken and Orlikoff, 2000. Studies: Peterson and Barney, 1952; Eguchi and Hirsh, 1969; Hillenbrand, Getty, Clark, and Wheeler, 1995; Lee, Potamianos, and Narayanan, 1999.

\section{Relationships among the vocal subsystems}

Although many studies have provided information regarding isolated subsystem function, much less is known about the interactive nature of the subsystem triad. Currently, much of the knowledge base regarding the details of vocal subsystem interaction has come from computer modeling of the vocal tract; however much of this data is primarily dedicated to the interactions of the laryngeal and resonance systems only ${ }^{50,54}$ A few studies have used respiratory kinematics, laryngeal aerodynamic measures, and acoustic measures in the form of overall SPL to simultaneously measure the three subsystems. ${ }^{33-35,55-57}$ Stathopoulos and Sapienza measured changes to the respiratory and laryngeal systems during variation of vocal intensity in males and 
females. ${ }^{33}$ Respiratory measures indicated that with increased loudness there was a general tendency for increased subglottic pressures. Women's airflow rates decreased, while males increased. Respiratory changes included a general trend for increased lung and ribcage volumes with increased vocal intensity with decreased abdominal movement. Women generally initiate speech at higher lung volumes than men. Of interest, this study reported that a few subjects did not follow the general trends in the study. The majority of subjects demonstrated that with increased loudness both respiratory kinematic movements and overall laryngeal resistance increased. A few subjects demonstrated a primarily respiratory adjustment characterized by increased lung volume initiations and excursions with a longer vocal fold open quotient. Another subset demonstrated a primarily laryngeal adaptation to increased loudness characterized by a short vocal fold open quotient with little change in the respiratory system.

Simultaneous measurements during whispering, have also demonstrated the interactive nature of changes in airflow and changes in respiratory kinematics. ${ }^{58}$ An unexpected finding of this study was that subjects did not inhale to higher lung volumes in response to the increased airflow through the glottis during whispering. Instead, lower lung volumes were observed on average.

One study has systematically perturbed oral pressures via pressure bleed (leak) by placing a tube on the oral cavity. ${ }^{35}$ It was demonstrated that during the oral pressure bleed that lung volume initiations and terminations were not significantly different, but terminations were different enough to reveal a significant differences in the length of lung volume excursions. This study did not reveal significant differences in men and women across conditions for lung volume measurements; however rib cage terminations were significantly lower for women. No significant differences in abdominal volumes were 
observed across conditions; however women were found to have smaller abdominal initiations than men. Aerodynamic measures indicated that subglottic pressure was decreased during the pressure bleed for both men and women. Average flow was decreased for both men and women. Acoustic measurements indicated no significant differences in overall SPL or in fundamental frequency across conditions for both men and women.

Another study used simultaneous measures to determine the strategies used by patients with Parkinson's Disease to increase vocal loudness during noise. ${ }^{59}$ Results indicated that while speaking in noise SPL was generally increased. The majority of patients demonstrated increased subglottic pressure when speaking in noise. Peak-topeak airflow rate was also generally increased. Lung volume initiations and terminations were generally increased in noise, but not lung volume excursions. This was also true for ribcage initiations, terminations and excursions. Abdominal initiations, terminations, and excursions followed the same pattern. Individual data revealed that patterning of the laryngeal and respiratory systems was highly individualized, despite the overall trends. A limitation to these studies is that the chosen measure of supraglottic activity, overall sound pressure level, does not permit insight into changes in the resonance strategies of the subjects and therefore does not permit insight into the interactions of all three subsystems.

When consideration is given to interactions among the vocal subsystems, it is also important to consider interactions within the individual subsystems and how they exert a pull on the overall patterning of the subsystem triad. A retrospective study by Gillespie et al., observed that during repeated syllable tasks, subjects with muscle tension dysphonia could be profiled into 5 different groups based on the relationships of estimated oral pressure and average airflow rates. Using cluster analyses, the authors 
were able to profile patients into groups based on distinct interactive patterns of pressure and airflow rate. Interestingly, greater than thirty percent fell into a profile which was within the established normative data range for these measures despite clinical diagnosis of muscle tension dysphonia. Data for this study did not directly observe the respiratory or resonance systems, however this study indicates that subsystem interactions are present both within and among the vocal subsystems and that a variety of combinations of pressure and airflow can characterize primary muscle tension dysphonia. $^{60}$

The current study was designed to expand on previous data by simultaneously measuring the three vocalization subsystems in a way that permitted descriptive characterization of the strategies used by individuals under both normal and perturbed voicing conditions. Based on previous literature and clinical applicability, we selected instrumentation that permitted measures that reflected the underlying physiologic mechanics that are useful to describe voice production. Using Dynamic Systems Theory as a guide, we hypothesized that individual differences would not be random, but rather would cluster around a central attractor state for both normal and disordered states. We anticipated that there would be differing strategies and attractor states across individuals both within the confines of normal as well as disordered voice production. We sought to descriptively characterize these important differences with the goal of highlighting important individual differences in phonatory regulation in order to better understand how individuals regulate phonation in different contexts. Understanding individual differences in phonatory regulation is envisioned to permit better understanding of normal and disordered voice production, characterize response to therapeutic interventions, and potentially provide predictive modeling power for more individualized treatments for 
specific voice disorders. Chapter three describes rationale and purpose of our perturbation design and describes the methodology used in this study. 


\section{CHAPTER 3: METHODS}

\section{Research Design and Methods}

This project used a repeated measures perturbation paradigm to study the relative contributions and interactions of the vocalization subsystems in response to a fictive laryngeal disorder of temporarily induced unilateral vocal fold paralysis (UVFP). This perturbation disrupted the valving action of the larynx resulting in increased airflow (leak) through the glottal valve. UVFP was chosen because the laryngeal valve is the gateway connecting the pressure source for phonation below the larynx and the resonance system above, representing a key site where pulmonary pressure is transduced into acoustic (oscillatory) airflow that excites the vocal tract. Disruption of this site permitted observation of the function of all three vocalization subsystems without the use of external devices that would preclude measurement of all three subsystems.

Temporary UVFP is a routinely used procedure in patients with adductor spasmodic dysphonia (ADSD) to determine candidacy for nerve sectioning, ${ }^{61-65}$ and has also been used to study the central representation of vocalization under fMRI conditions. ${ }^{66}$ We used this method to facilitate representative and simultaneous measurements for each subsystem during vocal tasks consisting of repeated sentences.

Measurements included acoustic formant spacing ${ }^{12}$ aerodynamic pressures and flows, ${ }^{67}$ and respiratory kinematic measures of the ribcage and abdomen. ${ }^{11}$ Data were collected in three conditions: 1) a non-perturbed condition (PRE), 2) paralysis condition (PAR), and 3) recovered condition (REC).

Because of the large number of measures and terms associated with this study, a list of terms has been provided for the reader's reference in Table 3.1. 


\section{Table 3.1}

\begin{tabular}{|c|c|}
\hline Term & Definition \\
\hline \multicolumn{2}{|l|}{ Respiratory Measures (Respiration) } \\
\hline Vital Capacity (VC) & $\begin{array}{l}\text { The amount of air exchanged during a maximal } \\
\text { inhalation and maximal expiration. Measured in } \\
\text { Liters. }\end{array}$ \\
\hline End Expiratory Level (EEL) & $\begin{array}{l}\text { The point of passive relaxation of the respiratory } \\
\text { system where no air is moving in or out of the } \\
\text { system. This is typically approximately } 35 \% \text { of a } \\
\text { subject's vital capacity. }\end{array}$ \\
\hline Lung Volume Initiation (LVI) & $\begin{array}{l}\text { The lung volume inhalation point prior to speech } \\
\text { initiation. Measured in percent of the total VC } \\
\text { and relative to EEL. }\end{array}$ \\
\hline Lung Volume Termination (LVT) & $\begin{array}{l}\text { The lung volume exhalation point where a } \\
\text { speech utterance is terminated. Measured in } \\
\% \mathrm{VC} \text { and relative to EEL. }\end{array}$ \\
\hline Lung Volume Excursion (LVT) & $\begin{array}{l}\text { The amount of VC used from LVI to LVE. } \\
\text { Calculated as LVI - LVT. }\end{array}$ \\
\hline Rib Cage Initiation ( $\mathrm{RCl})$ & $\begin{array}{l}\text { The maximal movement of the ribcage during } \\
\text { inhalation relative to EEL before the initiation of } \\
\text { speech. }\end{array}$ \\
\hline Rib Cage Termination (RCT) & $\begin{array}{l}\text { The point of ribcage movement measured at the } \\
\text { termination of a speech utterance relative to } \\
\text { EEL. }\end{array}$ \\
\hline Rib Cage Excursion (RCE) & $\begin{array}{l}\text { The total movement of the rib cage from } \mathrm{RCI} \text { to } \\
\mathrm{RCT} \text {. }\end{array}$ \\
\hline Abdominal Initiation $(\mathrm{ABI})$ & $\begin{array}{l}\text { The maximal movement of the abdomen during } \\
\text { inhalation relative to EEL before speech } \\
\text { initiation. }\end{array}$ \\
\hline Abdominal Termination (ABT) & $\begin{array}{l}\text { The point of abdominal movement measured at } \\
\text { the termination of a speech utterance relative to } \\
\text { EEL. }\end{array}$ \\
\hline Abdominal Excursion (ABE) & $\begin{array}{l}\text { The total movement of the abdomen from } A B I \text { to } \\
\text { ABT. }\end{array}$ \\
\hline \multicolumn{2}{|c|}{ Laryngeal Aerodynamic Measures (Phonation) } \\
\hline $\begin{array}{l}\text { Estimated Subglottic Air Pressure (Subglottic } \\
\text { Pressure }\left[\mathrm{P}_{\mathrm{s}}\right] \text { ) }\end{array}$ & $\begin{array}{l}\text { The amount of air pressure that builds up } \\
\text { underneath the vocal folds and glottis (space } \\
\text { between the vocal folds) during speech. This } \\
\text { measure is estimated from the oral pressure } \\
\text { during the consonant /p/ during a speech task. } \\
\text { Measured in cmH2O. }\end{array}$ \\
\hline Average Airflow Rate (Flow) & $\begin{array}{l}\text { The amount of air flowing between the vocal } \\
\text { folds during a speech task. Measured in liters per } \\
\text { second }(L / s) \text {. There is a theoretical inverse } \\
\text { relationship between } P_{s} \text { and flow. }\end{array}$ \\
\hline Vocal Tract Formant Measures (Resonance) & $\begin{array}{l}\text { Formants (F1, F2...etc.) are areas of enhanced } \\
\text { resonance within the vocal tract (throat cavity, } \\
\text { oral cavity, and nasal cavity) that act to filter the } \\
\text { sound generated from the vibrating vocal folds. } \\
\text { These areas give a boost to acoustic properties } \\
\text { of vocal output and reflect changes in the shape } \\
\text { of the vocal tract. Measured in Hertz (cycles per } \\
\text { second) and converted to the logarithmic "cent" } \\
\text { scale for direct comparisons between subjects. }\end{array}$ \\
\hline
\end{tabular}




\section{Subjects}

Ten healthy young adults with normal voices $(2 \mathrm{M} / 8 \mathrm{~F})$ volunteered for this study with ages ranging from 20 to 37 years (mean: 24, standard deviation [SD] 5.3). Subjects included university students and members of the community. Data were collected in a non-perturbed and a laryngeal perturbation condition (UVFP). Participant criteria for the study were as follows:

Inclusion Criteria:

1. Age $18-50$

2. English as native language

3. Perceptually normal speech, language, and voice

4. Non-smoking for at least the past 5 years

\section{Exclusion Criteria:}

1. Current or prior vocal pathology

2. Stroke or other neurological dysfunction

3. Neck or spinal surgery

4. Respiratory conditions (recent respiratory infections, asthma, paradoxical vocal fold movement)

5. Formal speaking or singing training

6. Allergies to Lidocaine, Epinephrine, or its derivatives

All procedures were approved by the University of Kentucky Institutional Review Board, and informed consent was obtained from all subjects. All assessments and measures were performed at the University of Kentucky Laryngeal \& Speech Dynamics Laboratory.

\section{Tasks and conditions}

Sentence task: Subjects produced ten repetitions of the sentence "Buy $\mathrm{Pa}$ or $\mathrm{Pa}$ a Pa Pa," adapted from Huber, ${ }^{59}$ at comfortable pitch and loudness levels, one time per breath group. A slow rate was used which was approximately $1-1.5$ syllables per 
second. The first and last two utterances from each group were not analyzed leaving the middle six trials from each group for analysis. This syllable train was produced in the following three conditions during the same session:

1. "Pre RLN block" (PRE): Subjects performed ten repetitions of the vocalization task at comfortable pitch and loudness.

2. "During RLN block" (PAR): After endoscopic verification of right vocal fold paralysis, subjects performed the identical vocalization task.

3. "Post RLN block" (REC): Subjects again performed the vocalization task after endoscopic conformation of recovered normal movement of the arytenoid complex.

Subjects were engaged in conversation with the experimenter for 1-2 minutes before measurements were taken in the PAR and REC conditions in order for the participant to adjust to the changes in sensory perception and voice quality. They were encouraged to speak "normally" in all conditions.

Because of potential resonances introduced by the non-vented mask, identical voicing conditions were repeated immediately in a mask-on and mask-off condition so that detailed formant information could be obtained. Data were analyzed in the mask-off condition as respiratory data were significantly different in some subjects in the mask-on condition. This was not expected as a study comparing vented and non-vented masks and their effect on respiratory measures did not demonstrate significant differences. ${ }^{38}$ Secondly, formants could not be determined in the mask-on condition due to distortion of the acoustic signal. Finally, because it is clinically assumed that oral airflow and subglottic pressure are the same with the mask-off, this condition was felt to be the appropriate condition to obtain the most accurate information. The mask-on/mask-off order was alternated between subjects to reduce potential order effects. 


\section{Measurement and Perturbation Techniques}

Vocal Fold Imaging: Subjects received a laryngeal exam via videostroboscopy to ensure normal vocal function prior to participation and two additional times during the study to document position of the paralyzed vocal fold, and also to document vocal fold recovery before the final set of voice measurements. Visual imaging of the vocal folds was performed using a laryngeal videostroboscopy system (Kay PENTAX RhinoLaryngeal Stroboscope - Model RLS 9100B) coupled to a 70-degree rigid scope (Kay PENTAX, Model SN 1541). Position of the paralytic vocal fold was rated (medial, paramedian, or abducted) by two speech-language pathologists with training in voice disorders not associated with the study. All subjects were judged to have a paramedian positioning with 100\% agreement between two different raters. Figure $\mathbf{3 . 1}$ and $\mathbf{3 . 2}$ compare vocal fold abduction during breathing, normal vocal fold closure during voicing, and paramedian paralysis during voicing. It can be seen that the vocal folds do not exhibit complete closure in the paralytic condition. This results in escape of air through the vocal fold valve.

\section{Figure 3.1}
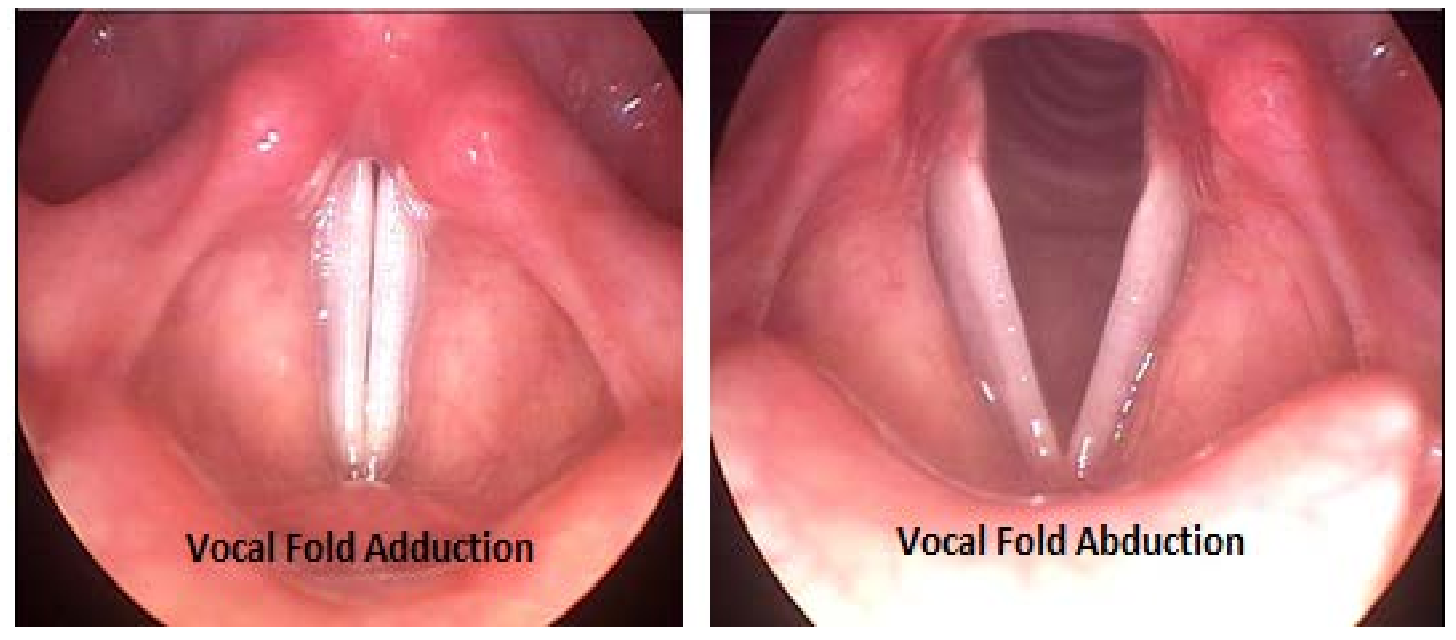
Figure 3.2
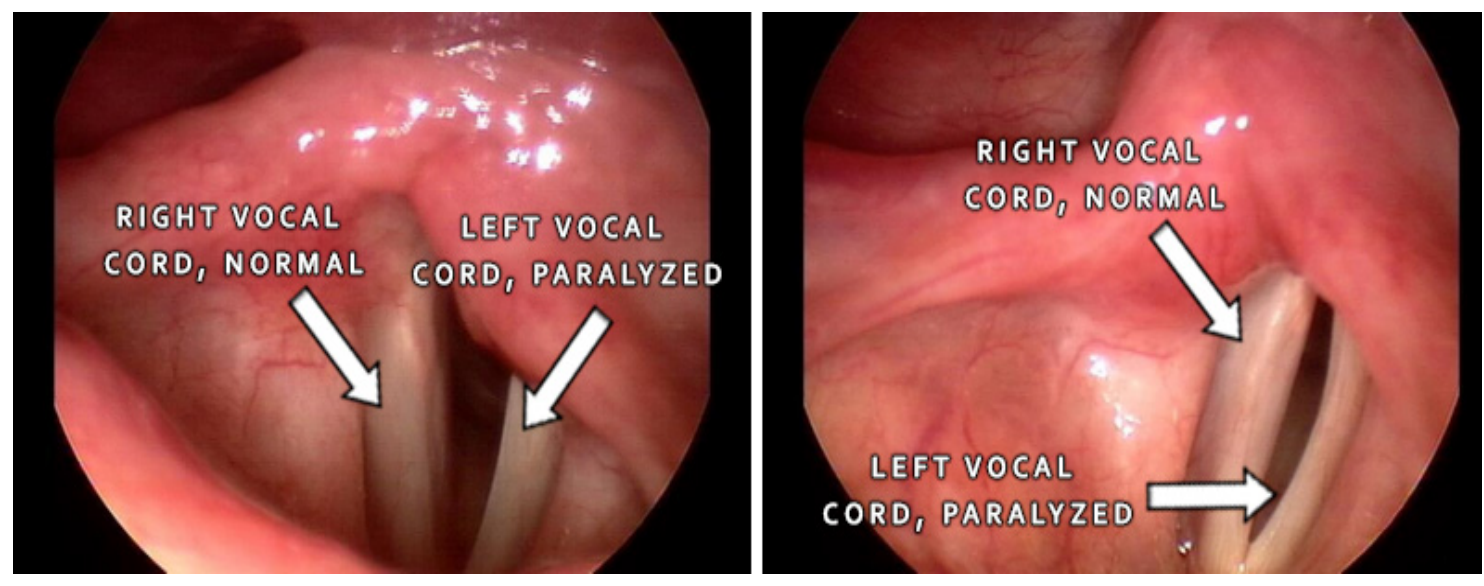

Respiratory Measurements: Respiratory Inductance Plethysmography was used to measure displacement of the chest wall which represents the lung volumes used during the voicing task, and also the individual contributions of the ribcage and abdomen to total chest wall movement. Respiratory data was transduced with the Inductotrace system (Ambulatory Monitoring) coupled to a Power Lab 16 channel A/D data acquisition system (AD Instruments 16/30) with data tracings displayed via Lab Chart software (ver. 7.2.5 AD Instruments). Data were stored digitally and analyzed in MATLAB (ver. 7.4, Mathworks, Inc).

Ribcage movement was transduced with the inductance band positioned around the circumference of the chest just below the axilla. The abdominal inductance band was placed around the abdomen at the level of the umbilicus just below the lowest floating rib. Ribcage and abdominal measures were taken relative to the subject's vital capacity as determined by spirometry to normalize results across subjects. The least squares method as described by Chadha, $1982^{22}$ was used to calibrate kinematic data.

For respiratory calibration, subjects completed 2 sessions of rest breathing into the spirometer for 45 seconds to determine their end-expiratory level (EEL). Subjects 
then participated in two sessions of "speech-like breathing" using the spirometer. This breathing pattern mimicked lung volumes more typical of speech to help with accuracy of the calibration procedure. Speech-like breathing was accomplished by having the subjects take and breath and silently "read in their mind" a sentence while exhaling. The sentence, "You buy Bobby a puppy now if he wants one," was read silently with the subject instructed to inhale, then "read" the sentence silently while exhaling, and breathing at the end of the sentence. The subject did this continually for two 45 second periods. Subjects then performed a series of maneuvers that reflect the minimum and maximum excursions of the ribcage and abdomen including a maximum inhalation and exhalation to determine vital capacity. To determine the rib cage and abdominal maximums and minimums, nose clips were placed on the subject so that no air could escape nasally. An oscilloscope was used to determine the EEL point. When the subject reached EEL during normal breathing, they were instructed to close their mouth and "suck their stomach in." A minimum of three trials were taken. The same process was applied to an additional movement where the subject was instructed to push their stomach out as much as possible. This was also completed a minimum of three times. These movements reflected the maximal movement of both the rib cage and abdomen.

The Moore-Penrose pseudo-inverse function was applied to the calibration data traces to determine the best fit of the chest wall movements as compared to spirometry measures with the least error. (Spirometry [total lung volume] $=(\mathrm{k})$ ribcage $+(\mathrm{k})$ abdomen) where $\mathrm{k}$ represents the non-absolute solution with the least squared error. Movements sensed by the respiratory bands were converted into representative lung volumes relative to the subject's vital capacity in order to normalize respiratory measurements. For the length of the study, subjects were seated in a comfortable, high 
backed chair (to reduce axial movements) reclined to 80 degrees with a foot rest to discourage leg crossing or excessive movement.

Figure 3.3 graphically illustrates lung volume initiations, terminations, and excursions. LVI is represented by the peak height on the left of the trace indicating the amount the subject inhales prior to starting the voicing task. LVT is represented by the lowest point (trough) on the right of the trace where the subject stops exhaling at the end of the voice task. The LVE is the distance between LVI and LVT and represents the amount of lung volume expired during the voice task. Lung volumes represent the sum of chest wall movement. Additionally, this total movement was broken down into the independent movement of the ribcage and abdomen to further characterize how subjects positioned the chest wall during voicing. Figure $\mathbf{3 . 4}$ demonstrates the respiratory band placement and seating of the subject during voice tasks.

Figure 3.3

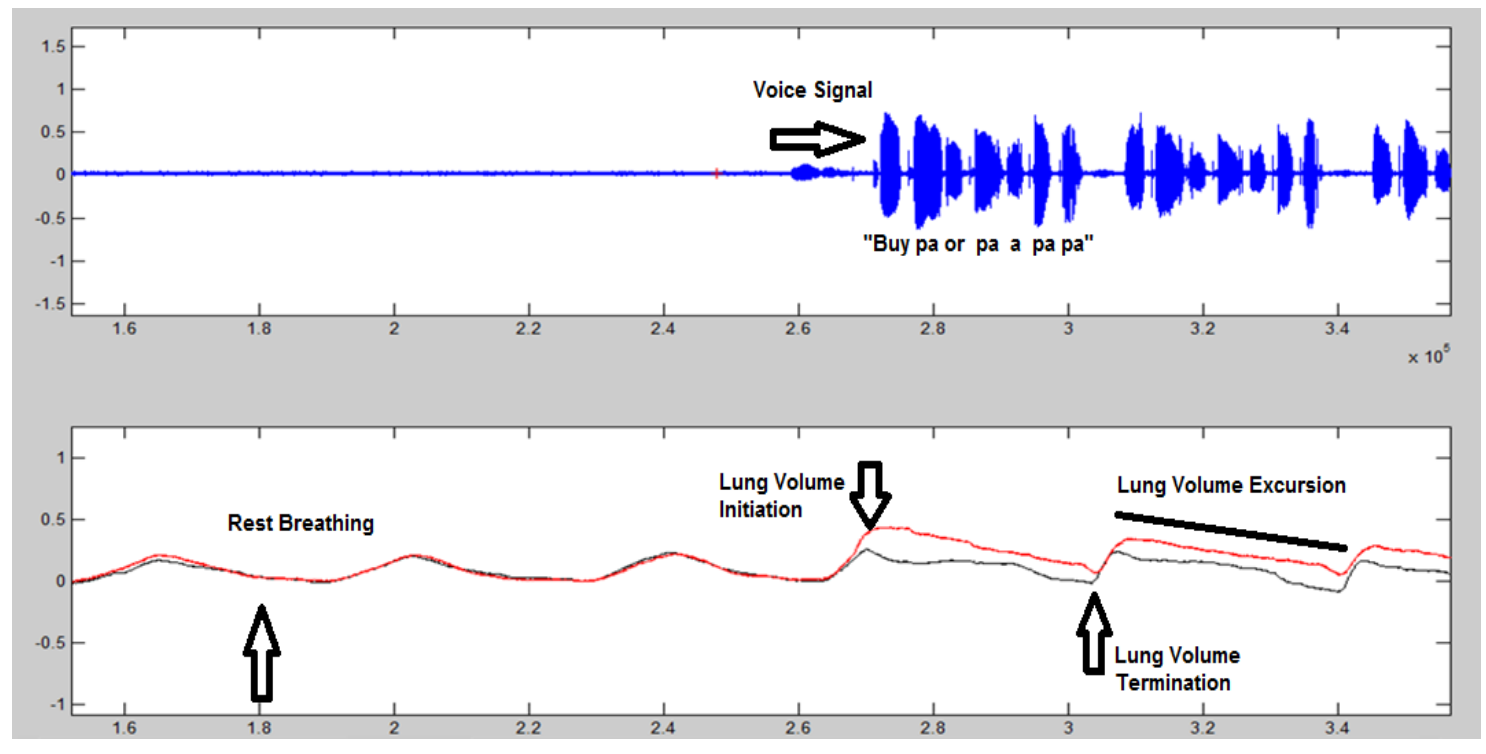

Aerodynamic Measurements: Real-time aerodynamic oral pressure and laryngeal airflow data were digitized and analyzed with computer software during production of the 
vocalization tasks. (Kay PENTAX Phonatory Aerodynamic System, Model 6000). Oral airflow data was collected via an anesthesia type mask placed over the nose and mouth directed to a pneumotachometer. A small flexible tube was inserted through the mask and positioned in the subject's mouth just behind the lips just behind the teeth to measure estimated subglottic pressure. The three accented syllables of "Buy $\underline{\mathrm{Pa}}$ or $\underline{\mathrm{Pa}} \mathrm{a}$ $\underline{\mathrm{Pa}} \mathrm{Pa}$ " were used for analysis, omitting the first, last, and unaccented syllables in the speech task. Figure $\mathbf{3 . 4}$ and $\mathbf{3 . 5}$ show the Phonatory Aerodynamic System and the pressure and airflow traces created during voice tasks. 
Figure 3.4

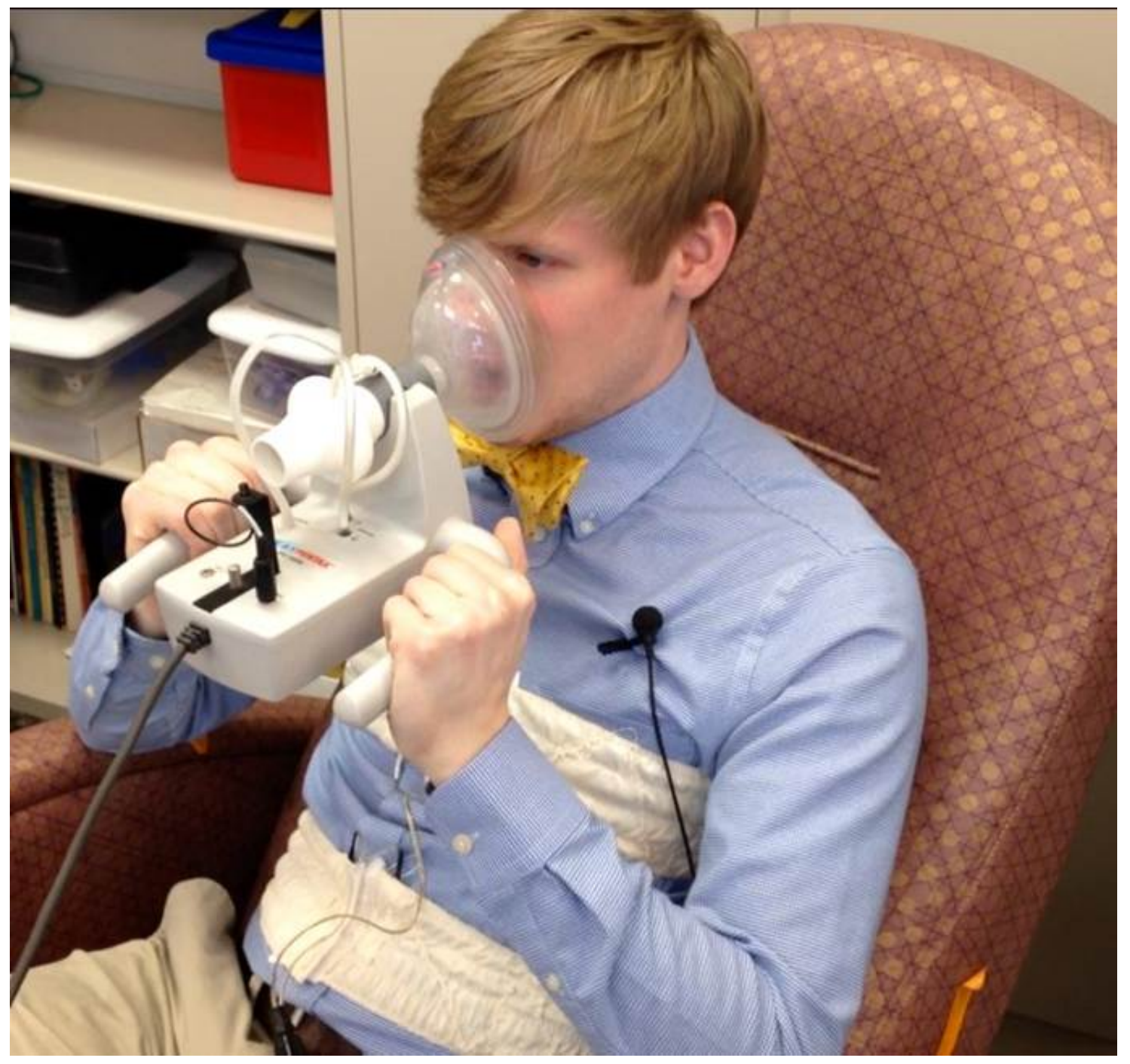


Figure 3.5

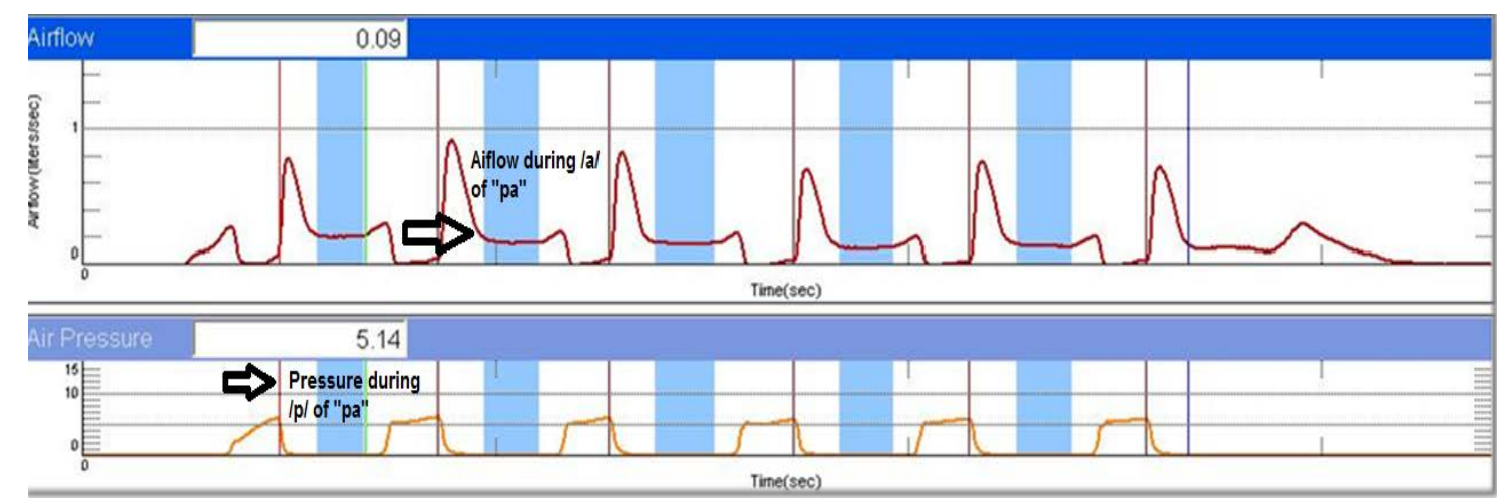

Acoustic Measurements: The voice tasks were transduced by a high quality condenser microphone (Shure SM-48 [mouth-to-microphone distance = 3 inches]). The voice signal was then analyzed by sound spectrography to determine characteristic resonance features of the voice acoustic signal via spacing of the first five formants. The same accented syllables of "Buy $\underline{\mathrm{Pa}}$ or $\underline{\mathrm{Pa}}$ a $\underline{\mathrm{Pa}} \mathrm{pa}$ " were used for analysis. Hertz (cycles per second) of the voice signal were converted into the logarithmic "cent" scale so that spacing between intervals were normalized and direct comparisons across individuals could be made. ${ }^{68}$ The "cent" scale was chosen because of the differences in fundamental frequency across subjects and between males and females. Because we were interested in the difference in spacing between vocal tract formants and not actual frequencies, conversion to the referenced and logarithmic cent scale functioned to standardize the distances between formants so that they were evenly scaled and directly comparable across subjects.

Acoustic data were digitally stored as .wav files and later analyzed using the Kay PENTAX CSL (Model 4500) Real-Time Spectrogram module (Model 5129, ver. 3.4.1) and with the Praat software package (Praat 5381). Figure 3.6 demonstrates the raw 
voice signal along with a spectrogram. The yellow lines on the spectrogram indicate the areas of formants $1-5$.

Figure 3.6

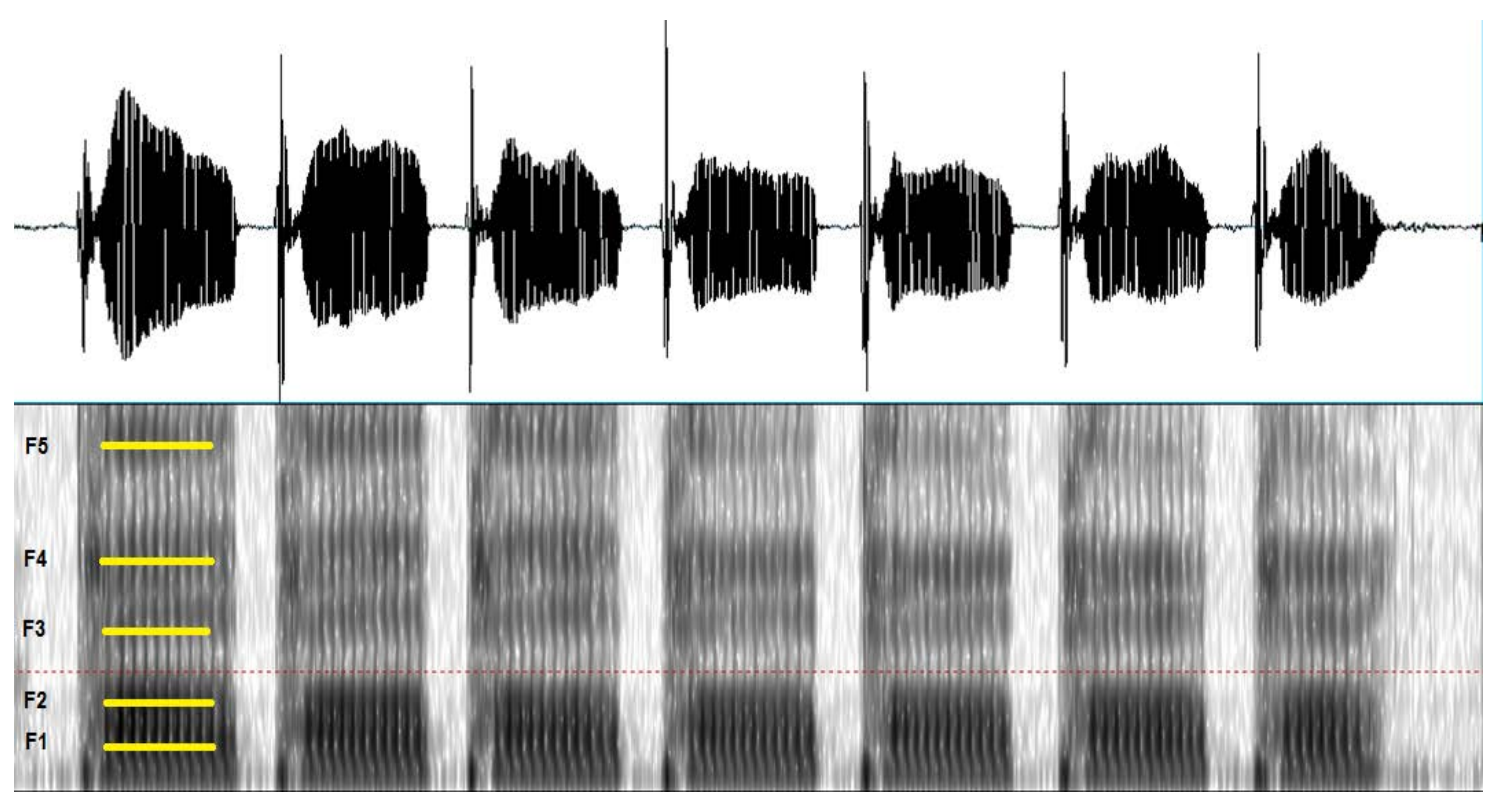

Laryngeal Perturbation Method: To produce a temporary UVFP for the

perturbation condition, the anterior neck tissue surrounding the right recurrent laryngeal nerve (RLN) was injected with a solution of $4-8 \mathrm{cc}$ of $2 \%$ lidocaine and diluted epinephrine 1:100,000 which produced a temporary (60-90 minute) nerve block to the muscles that control the affected vocal fold. This method was first described in $1976,{ }^{61}$ and has been used to assess candidacy for nerve sectioning in patients with Adductor Spasmodic Dysphonia, a focal dystonia which affects voice production. ${ }^{62,63,65}$ Recently lidocaine/epinephrine injections have been used to study its effects on muscle tension dysphonia and superior laryngeal nerve paralysis. ${ }^{64} \mathrm{~A}$ recent study has also used this method of temporary paralysis to study the central representation of vocalization on the brain using fMRI. ${ }^{66}$ Injections were performed by a board certified otolaryngologist with 25 years of experience in this procedure. Patients were monitored by the physician 
throughout data collection and until the nerve block dissipated. The nerve block functions to decrease the medial movement of the injected vocal fold and results in dysphonia and an air leak thereby perturbing the laryngeal subsystem due to lack of complete closure of the affected vocal fold. This perturbation is also clinically significant as unilateral vocal fold paralysis (UVFP) is a common vocal pathology. UVFP affects the patient's ability to effectively valve the airflow through the vocal folds during speech and results in a breathy, rough voice with increased pitch and decreased loudness making speech difficult to produce and perceptually difficult to hear and understand.

\section{Flow of Data Collection}

The flow of data collection is described briefly here to help the reader visualize the data collection process. First, after informed consent, subjects received the initial laryngeal exam to ensure normal vocal function. Subjects were then fitted with the respiratory bands to measure lung volume, rib cage, and abdomen kinematics. Subjects were then seated in the reclining chair and spirometry was performed to determine the subject's vital capacity and calibrate the respiratory band movement into a lung volume index representing the volumes used during the speech task. Maneuvers designed to measure the minimum and maximum excursion of the rib cage and abdomen were also performed a minimum of three times to calibrate respiratory band movement into the proportional contributions of the rib cage and abdomen during voice tasks. Once the respiratory bands were calibrated to the subject, voice tasks were performed into the laryngeal mask for aerodynamic measures, and then again into the microphone for acoustic measures.

After the initial measurement session, the physician then administered the lidocaine injection for the paralysis condition. When the subject was audibly dysphonic, a 
laryngeal exam was then performed again to document the right true vocal fold paralysis. After confirmation, the PI engaged the participant in conversation for 2-3 minutes so that the subject had to use the new voice in conversation. The acoustic, aerodynamic, and respiratory measures were then re-taken. After the subject's voice returned to baseline, a third laryngeal exam was performed to demonstrate that the affected vocal fold had returned to full movement. The subject was again engaged in conversation for 2-3 minutes to permit use of the new voice quality. Acoustic, aerodynamic, and respiratory measures were then taken a third time in the recovery condition.

\section{Data Analysis}

Data were analyzed using JMP (Cary, NC, USA) by SAS ver. 11. Descriptive statistics (means and SD) were calculated for all subjects. Group differences across task conditions were assessed with repeated-measures ANOVA. Correlations with $r^{2}$ values were calculated for change scores on all dependent variables with airflow rate (flow) serving as the explanatory variable to determine how each dependent variable changed due to increased airflow rate in the perturbation condition. Change scores were also standardized by subtracting the mean and dividing by the standard deviation for all independent variables to determine which variables were most influential to the changes between conditions.

Chapter 4 presents the results of our study first in terms of group data and also by subject to highlight the important differences that cannot readily be viewed by examination of the group data. 


\section{CHAPTER 4: RESULTS}

This chapter presents the main results of the group data for the study. Additional correlational analyses, and detailed analyses of the data by individual, are presented in Appendices 1, \& 2. Discussion of the group and individual data are synthesized in Chapter 5.

Demographic data for all subjects is presented in Table 4.1 Descriptive statistics (means and standard deviations) for all dependent variables for the PRE, PAR, and REC conditions are presented in Table 4.3.

\section{Table 4.1}

Demographic data for subject and age in years

\begin{tabular}{ll}
\hline Subject \# & Age (years) \\
\hline 01_F & 22 \\
02_F & 25 \\
03_F & 22 \\
04_M & 29 \\
05_F & 24 \\
06_M & 37 \\
07_F & 21 \\
08_F & 21 \\
09_F & 20 \\
10_F & 20 \\
\hline
\end{tabular}




\section{Reliability}

To establish inter-measurer reliability, data from two subjects (1 male and 1 female) were randomly selected for remeasurement. Aerodynamic measures were not remeasured as they are completely automated by the KayPENTAX Phonatory Aerodynamic System. Formant measurements and respiratory kinematics require manual selection of data points and therefore reliability analysis was only applied to these measures. Intraclass Correlation Coefficients (ICC) using a 2-factor mixed random effect model were calculated on the two sets of measurements. ICC values were high between raters indicating excellent reliability. Interpretation for the ICC values were determined as follows: $<0.40=$ poor reliability, $0.40-0.75=$ fair to good reliability, and $>0.75=$ excellent reliability. ${ }^{69,70}$ Results are reported in Table 4.2.

\section{Table 4.2}

\begin{tabular}{lll}
\hline Intraclass Correlation Coefficients (ICC) with 95\% Confidence Intervals (Cl) \\
Measure & ICC Value & $95 \% \mathbf{C l}$ \\
\hline Formant (F5) & .953 & $.713-.993$ \\
Formant (F4) & .855 & $.136-.979$ \\
\hline Formant (F3) & .994 & $-4.28-.677$ \\
Formant (F2) & .947 & $.680-.992$ \\
Formant (F1) & .785 & $-1.086-.971$ \\
Lung Volume Initiation (LVI) & .992 & $.375-.999$ \\
Lung Volume Termination (LVT) & .992 & $.782-.999$ \\
Lung Volume Excursion (LVE) & .864 & $-.150-.983$ \\
Rib Cage Initiation (RCI) & .998 & $.785-1.00$ \\
Rib Cage Termination (RCT) & .998 & $.987-1.00$ \\
Rib Cage Excursion (RCE) & .987 & $.923-.998$ \\
Abdominal Initiation (ABI) & .959 & $.537-.995$ \\
Abdominal Termination (ABT) & .938 & $-0.58-.993$ \\
Abdominal Excursion (ABE) & .744 & $-2.12-.964$ \\
\hline
\end{tabular}




\section{Table 4.3}

Repeated Measures ANOVA - Descriptive Statistics. Group Means, Standard Deviations, and Ranges by Condition (PRE, PAR, REC)

\begin{tabular}{|c|c|c|c|c|}
\hline Measures & Condition & Mean & (SD) & Range \\
\hline \multicolumn{5}{|c|}{ Acoustic measures - Distance between formants in "cents" } \\
\hline \multirow[t]{3}{*}{ F5-F4 } & PRE & 496.7 & (135.96) & $218-680$ \\
\hline & PAR & 423.4 & (99.37) & $232-586$ \\
\hline & REC & 537 & (146.28) & $279-689$ \\
\hline \multirow[t]{3}{*}{ F4-F3 } & PRE & 591.2 & (106.48) & $430-742$ \\
\hline & PAR & 621 & $(80.42)$ & $525-778$ \\
\hline & REC & 591.6 & $(84.31)$ & $460-710$ \\
\hline \multirow[t]{3}{*}{ F3-F2 } & PRE & 1570.2 & (167.85) & $1354-1859$ \\
\hline & PAR & 1533 & $(262.86)$ & $1002-1870$ \\
\hline & REC & 1504.5 & $(160.62)$ & $1343-1847$ \\
\hline \multirow[t]{3}{*}{ F2-F1 } & PRE & 739.8 & (134.02) & $548-947$ \\
\hline & PAR & 1183.7 & (259.39) & $845-1601$ \\
\hline & REC & 640.6 & (196.99) & $239-911$ \\
\hline \multicolumn{5}{|c|}{$\begin{array}{l}\text { Aerodynamic Measures - Estimated Subglottic Pressure in centimeters of water (Ps in cmH2O), } \\
\text { Mean Peak Airflow in Liters per Second (Flow in L/s) }\end{array}$} \\
\hline \multirow[t]{3}{*}{ Ps } & PRE & 6.93 & (1.59) & $4.69-8.82$ \\
\hline & PAR & 6.92 & (1.98) & $3.81-10.09$ \\
\hline & REC & 7.77 & $(2.2)$ & $4.98-12.29$ \\
\hline \multirow[t]{3}{*}{ Flow } & PRE & 0.10 & $(0.06)$ & $0.02-0.23$ \\
\hline & PAR & 0.20 & $(0.12)$ & $0.06-0.47$ \\
\hline & REC & 0.11 & (0.07) & $0.03-0.22$ \\
\hline
\end{tabular}

Respiratory Measures - Lung Volume Initiation, Termination, and Excursion: (LVI, LVT, LVE), Rib Cage Initiation, Termination, and Excursion: (RCI, RCT, RCE), and Abdominal Initiation,

Termination, and Excursion: ( $A B I, A B T, A B E)$ as \% of Vital Capacity and Relative to End Expiratory Level (\%VC relative to EEL)

\begin{tabular}{llll} 
LVI & PRE & $30.93(8.81)$ & $15.66-43.98$ \\
& PAR & $24.17(11.98)$ & $5.4-43.14$ \\
LVT & REC & $22.72(12.29)$ & $5.84-40.25$ \\
& PRE & $19.40(10.44)$ & $1.77-33.28$ \\
LVE & PAR & $8.46(17.05)$ & $-16.45-33.1$ \\
& REC & $9.59(13.66)$ & $-8.53-27.81$ \\
\hline RCI & PRE & $11.54(3.96)$ & $6.38-19.51$ \\
& PAR & $15.71(6.16)$ & $10.03-29.17$ \\
& REC & $13.13(3.99)$ & $8.94-22.54$ \\
\hline RCT & PRE & $32.33(12.90)$ & $14.47-55.92$ \\
& PAR & $26.19(14.83)$ & $6.1-57.59$ \\
& REC & $23.30(15.13)$ & $0.57-47.27$ \\
RCE & PRE & $22.93(14.67)$ & $6.95-51.02$ \\
& PAR & $12.53(18.48)$ & $-9.56-51.05$ \\
& REC & $13.76(15.83)$ & $-7.63-41.05$ \\
\hline ABI & PRE & $9.41(4.31)$ & $4.33-15.89$ \\
& PAR & $13.66(6.83)$ & $6.53-30.7$ \\
& REC & $9.54(2.77)$ & $6.21-15.9$ \\
ABT & PRE & $19.17(7.10)$ & $5.69-28.81$ \\
& PAR & $12.64(9.91)$ & $-2.17-29.91$ \\
& REC & $13.78(8.97)$ & $2.52-27.83$ \\
\hline ABE & PRE & $9.44(7.22)$ & $-2.48-21.24$ \\
& PAR & $-1.05(16.63)$ & $-35-77-17.94$ \\
& REC & $1.40(10.05)$ & $-14.63-13.62$ \\
\hline
\end{tabular}




\section{Group Data Results}

Results from the repeated measures ANOVA for group data by condition (PRE, PAR, REC) are reported for each dependent variable by subsystem: acoustic measures, aerodynamic measures, and respiratory measures in Table 4.4. Post-hoc Tukey's Honestly Significant Difference testing was applied to determine significant differences between conditions. Significant differences between the PRE and PAR conditions are described below. 


\section{Table 4.4}

Repeated Measures ANOVA. Tukey's Honestly Significant Difference test was applied to determine significant differences between conditions. ${ }^{* *} \mathrm{p}<0.05$.

\begin{tabular}{lll}
\hline Measures & $\begin{array}{l}\text { F value }(p) \\
(d f=2)\end{array}$ & Condition
\end{tabular}

\section{Acoustic Measures}

\begin{tabular}{|c|c|c|}
\hline F5-F4 & $4.80(0.021)^{\star \star}$ & $\begin{array}{l}\text { REC - PAR } p=0.018^{\star} \\
\text { PRE - PAR } p=0.148 \\
\text { REC - PRE } p=0.054\end{array}$ \\
\hline F4-F3 & $2.72(0.093)$ & $\begin{array}{l}\text { PAR - PRE } p=0.133 \\
\text { PAR - REC } p=0.139 \\
\text { REC - PRE } p=0.999\end{array}$ \\
\hline F3-F2 & $0.72(0.499)$ & $\begin{array}{l}\text { PRE - REC } p=0.468 \\
\text { PRE - PAR } p=0.778 \\
\text { PAR - REC } p=0.862\end{array}$ \\
\hline F2-F1 & $17.57(<0.0001)^{\star \star}$ & $\begin{array}{l}\text { PAR - REC } p=<0.0001^{*} \\
\text { PAR - PRE } p=0.0007^{\star} \\
\text { PRE - REC } p=0.576\end{array}$ \\
\hline \multicolumn{3}{|c|}{ Aerodynamic Measures } \\
\hline Ps & $1.56(0.236)$ & $\begin{array}{l}\text { REC - PAR } p=0.294 \\
\text { REC - PRE } p=0.305 \\
\text { PER-PAR } p=0.999\end{array}$ \\
\hline Flow & $7.38(0.0046)^{\star \star}$ & $\begin{array}{l}\text { PAR - PRE } p=0.0078^{*} \\
\text { PAR - REC } p=0.0131^{*} \\
\text { REC - PRE } p=0.968\end{array}$ \\
\hline
\end{tabular}

Respiratory Measures

\begin{tabular}{|c|c|c|}
\hline LVI & $7.79(0.0036)^{\star \star}$ & $\begin{array}{l}\text { PRE - REC } p=0.004^{*} \\
\text { PRE - PAR } p=0.018^{\star} \\
\text { PAR - REC } p=0.792\end{array}$ \\
\hline LVT & $6.66(0.0068)^{\star \star}$ & $\begin{array}{l}\text { PRE - PAR } p=0.010^{\star} \\
\text { PRE - REC } p=0.021^{\star} \\
\text { REC - PAR } p=0.938\end{array}$ \\
\hline LVE & 3.12 (0.069) & $\begin{array}{l}\text { PAR - PRE } p=0.058 \\
\text { PAR - REC } p=0.302 \\
\text { REC - PRE } p=0.618\end{array}$ \\
\hline $\mathrm{RCl}$ & $7.68(0.0039)^{\star \star}$ & $\begin{array}{l}\text { PRE - REC } p=0.003^{\star} \\
\text { PRE - PAR } p=0.045^{\star} \\
\text { PAR - REC } p=0.452\end{array}$ \\
\hline RCT & $8.37(0.0027)^{\star \star}$ & $\begin{array}{l}\text { PRE - PAR } p=0.004^{\star} \\
\text { PRE - REC } p=0.012^{*} \\
\text { REC - PAR } p=0.898\end{array}$ \\
\hline RCE & $4.79(0.0215)^{\star \star}$ & $\begin{array}{l}\text { PAR - PRE } p=0.036^{*} \\
\text { PAR - REC } p=0.042^{\star} \\
\text { REC - PRE } p=0.996\end{array}$ \\
\hline $\mathrm{ABI}$ & $4.11(0.0339)^{\star \star}$ & $\begin{array}{l}\text { PRE - PAR } p=0.038^{*} \\
\text { PRE - REC } p=0.096 \\
\text { REC - PAR } p=0.887\end{array}$ \\
\hline ABT & 3.28 (0.061) & $\begin{array}{l}\text { PRE - PAR } p=0.061 \\
\text { PRE - REC } p=0.174 \\
\text { REC - PAR } p=0.837\end{array}$ \\
\hline ABE & $1.68(0.215)$ & $\begin{array}{l}\text { PAR - PRE } p=0.199 \\
\text { REC - PRE } p=0.466 \\
\text { PAR - REC } p=0.827\end{array}$ \\
\hline
\end{tabular}


Acoustic Measures Summary: Formant spacing (F1 - F5 in "cents")

1. F2 - F1 spacing was significantly increased from PRE to PAR (mean change: 444 cents), $[p=0.0007]$

2. F3 - F2 spacing was not significantly different, but decreased on average from PRE to PAR (mean change: -37 cents), $[p=0.778]$.

3. F4 - F3 spacing was not significantly different, but increased on average from PRE to PAR (mean change: 30 cents), [p $=0.133]$.

4. F5 - F4 spacing was not significantly different from PRE to PAR, but tended to decrease (mean change: 73 cents), $[p=0.148]$. The decreasing trend in spacing was primarily a result from a rising of F4 noted in all subjects in the PAR condition.

Additionally, to determine if associations existed in the spatial relationships between formants, linear regression was performed on the change scores from the PRE to PAR conditions. There was a significant association between F5 - F4 and F2 - F1 indicating that as the space between F2 - F1 increased, the space between F5 - F4 decreased, $\left(r^{2}=0.63\right)$. There was also a significant association between F4 - F3 and F2 - F1 indicating that as F2 - F1 increased F4 - F3 decreased. $\left(r^{2}=0.49\right)$.

\section{Aerodynamic Measures Summary:}

1. Estimated subglottic pressure $\left(P_{s}\right.$ in $\left.\mathrm{cmH} 20\right)$. There were no significant differences in $P_{\mathrm{s}}$ across the three conditions $[F(2)=0.236]$.

2. Mean peak airflow rate (Flow in $\mathrm{mL} / \mathrm{s})$. Flow was significantly increased from the PRE to PAR condition (mean change: $100 \mathrm{~mL} / \mathrm{s}),[F(2)=0.0046(p=0.008)]$. 


\section{Respiratory Measures Summary:}

1. Lung Volume Initiation (LVI). LVI was significantly decreased in the PRE to PAR condition $\quad[p=0.018]$, (mean change: $-6.76 \% \mathrm{VC}$ ).

2. Lung Volume Termination (LVT). LVT was significantly decreased in the PRE to PAR condition $[p=0.010]$ (mean change: $-10.94 \%$ VC).

3. Lung Volume Excursion (LVE). There was no overall difference between conditions for LVE $[F(2)=0.0687]$.

4. Ribcage Initiation $(\mathrm{RCl})$. $\mathrm{RCI}$ was significantly decreased from the PRE to PAR condition [ $\mathrm{p}=0.045]$, (mean change: -6.14$)$.

5. Ribcage Termination (RCT) RCT was significantly decreased from PRE to PAR [p = 0.004], (mean change: -10.4).

6. Ribcage Excursion (RCE). RCE significantly increased from the PRE to PAR condition. $[p=0.036]$, (mean change: 4.25$)$.

7. Abdominal Initiation ( $A B I)$. ABI significantly decreased from PRE to PAR condition. [p $=0.038]$, (mean change: 6.53$)$.

8. Abdominal Termination (ABT). There was no overall difference between conditions for ABT, $[F(2)=0.0610]$.

9. Abdominal Excursion (ABE). There was no overall significant difference between conditions for $\mathrm{ABE},[\mathrm{F}(2)=0.2151]$.

Chapter 5 presents a discussion of the individual and group data along with limitations, delimitations and future directions for this line of study. 


\section{CHAPTER 5: DISCUSSION}

This study was designed to examine the changes to and interactions of the proportional contributions of respiration, phonation, and resonance in response to a perturbation to the laryngeal valve via a temporary unilateral vocal fold paralysis. This perturbation created an increase in airflow secondary to a decrease in vocal fold closure. The laryngeal valve was chosen for perturbation for two reasons: 1) glottic incompetence, whether neurogenic or due to presence of vocal fold lesion is a common clinical presentation that causes a decrease in laryngeal airway resistance and therefore requires adjustment of the subsystems to compensate for increased escaping air through the glottis, and 2) it was important to perturb the system in such a way to leave the oral cavity unaffected in order to obtain resonance measures. Previous studies have not measured the contribution of the resonance system in such a way to make inferences regarding the changing physiology of the vocal tract. This was an important expansion of this study. This study also was conducted to expand on previous research which has simultaneously measured the three subsystems of phonation.

An additional goal of this study was to characterize individual regulation and adaptation of the subsystems of phonation in both normal and perturbed states. Individual differences are often described clinically, however it was important to describe and quantify these differences to begin to determine causal relationships of these changes. It was apparent that average data, while important, blurred essential information regarding individual performance. Some individuals did not follow the group means and it is important to view these differences, not as error, but as true and meaningful differences in adaptation. This study intentionally loosened internal control criteria because external validity was of interest. For example, it was acknowledged that the degree of air leak in the paralytic condition would not be the same for all subjects 
and therefore was a lack of experimental control. Voice tasks were also not controlled for frequency and intensity because it was of interest to observe how individuals respond to perturbation without artificial constraints. This may be viewed as a methodological weakness; however this study was planned with the understanding that previous work has demonstrated greater reliability under more controlled conditions, ${ }^{16}$ and those data are used widely as clinical normative standards. Practical and clinically useful data require attention to both internal and external validity and this study was intentionally designed with the idea that even though clinical patients are similar in diagnoses, they may differ widely in their vocal management strategies. Understanding the physiologic variability among individuals was deemed crucial in order to make useful interpretation of the data to develop more personalized treatment for patients with voice disorders.

We used dynamic systems theory as a framework to guide our experimental thought and design of this study. ${ }^{13}$ It was clear from the data that a solely reductive approach to data analysis left gaps in description of how the three subsystems of phonation regulate themselves in individuals as no single target solution to subsystem regulation was observed. Because true linear systems do not exist in human beings, we thought it important to observe the entire system, not by separating its components, but rather by observing how the component parts work together. Although there were individual differences, this methodology helped to determine which variables were similar (and different) across individuals in a more meaningful way than simple averaging. 


\section{Group Data}

\section{Acoustic Measures - Resonance characteristics}

There were decreases in spacing between F5 - F4 and significant increases in the spacing between F2 - F1 in response to increased airflow in the perturbation condition. F5 - F4 and F2 - F1 were noted to covary during the paralysis condition and were significantly correlated. F3 - F2 decreased on average, but not significantly so. These changes in formant spacing reflect physiologic alterations in the vocal tract. ${ }^{43}$ All subjects demonstrated a degree of supraglottic squeeze (narrowing) on endoscopy during perturbation, likely in attempt to maintain subglottic pressure and reduce the increased airflow by closing the glottic gap during phonation.

The area immediately above the larynx known as the epilaryx tube has been associated with a clustering of formants F5 - F3 when it is either narrowed, or when the pharynx above it is expanded (effectively narrowing the epilaryx space) ${ }^{51}$ This is well known in the singing voice as the singer's formant cluster and has been demonstrated to increase the acoustic output in the $25 \mathrm{kHz}-35 \mathrm{kHz}$ range which is in the area for

formants F3- F5. ${ }^{45,71}$ Increased upper harmonic boosts in this area has also been observed by Guzman et al., in the speaking voice after altering the epilarynx and pharyngeal area after use of straw/tube phonation. ${ }^{52}$ Effective narrowing of the epilarynx tube is a commonly used strategy by singers and speakers to project their voices by taking advantage of the increased resonance (intensity) instead of resorting to straining to increase vocal intensity.

It is likely that epilaryngeal narrowing noted in this study was the result of increased supraglottic activity in order to compensate for the loss of pressure and increase in airflow resulting from increased glottic incompetence. The changes in 
formant spacing likely reflect epilarynx narrowing (decreasing the overall spacing among F5 - F3) and pharyngeal widening (increasing the distance between F2 - F1). It is unclear if this is an attempt to simply maintain pressure and airflow, or if this represents a conscious attempt to boost acoustic output power as a result of sensory modification during the paralysis condition. It seems more plausible that the formant changes are simply the acoustic result of an attempt to maintain laryngeal airway resistance during perturbation. This idea was corroborated by the fact that airflow increased significantly, subglottic pressure was generally maintained, and there was a more active muscular response from the respiratory system in response to the laryngeal perturbation.

\section{Aerodynamic Measures}

Estimated Subglottic Pressure $\left(\boldsymbol{P}_{\mathrm{s}}\right)$ : $\mathrm{P}_{\mathrm{s}}$ was not significantly changed on average in the perturbation condition despite increased glottic incompetence with increased airflow. It is known that for greater glottic air leak, increased $\mathrm{P}_{\mathrm{s}}$ must occur in order to maintain laryngeal airway resistance. Sapienza and Stathopoulos studied women with vocal nodules compared with matched controls and noted that the nodule group on average did not have significantly different pressure rates despite increased airflow rates. ${ }^{72}$ They did observe that the nodule group tended to inhale to lower lung volumes, had more negative lung volume terminations and longer lung volume excursions. This indicated that there was an active respiratory response in order to maintain subglottic pressure in the nodule group. This phenomenon has also been observed during whispering indicating that subjects did not use respiratory recoil forces to their advantage during whispered speech. ${ }^{58}$

Similarly, $P_{s}$ in this study was maintained in response to decreased laryngeal airway resistance. Overall voice sound pressure level (dB SPL) was also not significantly 
different between conditions [PRE $73.95 \mathrm{~dB}$ (1.92), PAR $72.1 \mathrm{~dB}(2.30), \mathrm{p}=0.084$ ] indicating that subjects did not speak significantly louder or softer during paralysis. Because there is a known linear relationship between $\mathrm{P}_{\mathrm{s}}$ and $\mathrm{SPL},{ }^{72}$ maintenance of $\mathrm{dB}$ level corroborated the maintenance of $\mathrm{P}_{\mathrm{s}}$

\section{Airflow Rate}

Airflow rate was significantly different between conditions. This was expected secondary to varying degrees of glottic incompetence during the paralysis condition creating an air leak through the glottis. All subjects were judged to have paramedian (off the midline) paralysis during perturbation. The variable airflow rates demonstrate that the response to a similar physiologic configuration of glottic incompetence results in very different airflow rate responses across individuals as flow rates varied from $60-470$ $\mathrm{mL} / \mathrm{s}$ across subjects.

\section{Respiratory Measures}

Group data demonstrated that in order to compensate for increased airflow in the perturbation condition, subjects generally decreased their lung volume inhalation, and had lower lung volume terminations. Subjects did not inhale to higher lung volumes to take advantage of respiratory recoil forces, but rather oppositely inhaled less and used active muscle involvement during the expiratory limb to maintain subglottic pressure. Lung volume excursions were longer indicating a loss of laryngeal airway resistance during speech tasks. Similarly, rib cage $(R C)$ and abdominal $(A B)$ measures both demonstrated lower initiations, more negative terminations, and longer excursions. The average data reflect a more active use of the respiratory system during exhalation in response to increased airflow in order to maintain pressure for speech. The respiratory strategy used by the majority of the subjects in this study is counterintuitive in regard to 
respiratory initiations as it would seem more efficient to increase inhalation to take advantage of passive respiratory recoil forces; however similar strategies of smaller inhalations have been noted in patients with vocal nodules and during whispering. ${ }^{58,72}$ One other perturbation study using an oral pressure bleed also did not observe significantly higher lung volume initiations during perturbation to compensate to a loss of oral pressure ${ }^{35}$ however similar to this study, respiratory terminations were generally lower and respiratory excursions were generally greater. It is unclear why this respiratory strategy appears to result from a decrease in laryngeal airway resistance. One possibility is an active respiratory response to a change in sensory feedback. Because the body prefers to work in gestalt, it may be that the laryngeal compensatory hyperfunction resulting in attempt to increase laryngeal airway resistance may also be accompanied by more active respiratory drive from resulting sensory changes in the laryngeal subsystem. Stated differently, subjects may feel the need to "do something" extra in order to maintain the usual sensation of respiratory support during voicing.

\section{Individual Data}

Important observations regarding individual variability are not apparent in the averaged data. A secondary aim of this project was to examine the individual differences in the proportional contributions of respiration, phonation, and resonance during vocalization. This is important as it was demonstrated that a paramedian paralysis yielded quite different results in terms of airflow rate, and in individuals' responses to the laryngeal perturbation. In order to better understand the ways in which individuals cope with a loss of laryngeal airway resistance, the individual changes during perturbation are of importance. Individual differences in phonatory strategies will be discussed in terms of differences and similarities across individuals. 
Subjects 01, 08, 09, and 10 demonstrated similar respiratory patterns during the PAR condition. All of these subjects had RC and $A B$ initiations and terminations above EEL before and during perturbation. With the exception of the small increase in RC initiation in subject 09, the remainder had lower initiations, and more negative terminations. Because displacement of the $\mathrm{RC}$ and $\mathrm{AB}$ components remained in the upper right quadrant of the kinematic plot (above EEL for both $R C$ and $A B$ ) this indicated a passive response strategy of the respiratory mechanism before perturbation and also in response to the vocal fold paralysis.

Subjects 01 , and 08 increased $P_{s}$ similarly by 1.41 and $1.29 \mathrm{cmH} 20$ respectively in the PAR condition. Despite similar $\mathrm{P}_{\mathrm{s}}$, subject 01 had a flow increase of $80 \%$ while subject 08 only had a $6 \%$ increase in flow. The distance between F5 - F4 decreased and the space between F2 - F1 increased similarly in these two subjects.

Overall, these two subjects demonstrated strong changes in supraglottic activity and a lesser respiratory response to perturbation.

Subject 09 decreased $\mathrm{P}_{\mathrm{s}}$ by $8 \%$ and increased flow by $60 \%$. F5 - F4 increased by $10 \%$ and F2 - F1 increased by $16 \%$. Subject 09 was the only one to mildly increase $\mathrm{RC}$ initiation. Overall, this subject's strategy indicated a respiratory strategy that took more advantage of passive recoil forces to maintain pressure. Flow rates changed by 40 $\mathrm{mL} / \mathrm{s}$ and there was little response from the supralaryngeal component.

Subject 10 had the highest $\mathrm{RC}$ and $\mathrm{AB}$ initiations and terminations of the group. Pressure and flow were highly altered during perturbation for this subject $(-3.63 \mathrm{cmH} 20$ and $160 \mathrm{~mL} / \mathrm{s}) . \mathrm{RC}$ and $\mathrm{AB}$ terminations decreased indicating increased respiratory drive, but this was more passively provided as the initiations and terminations were well above EEL (seen in the upper right quadrant of the kinematic plot). 
Overall, these four subjects, although through slightly different means, employed a more passive respiratory strategy with stronger changes noted in the supralaryngeal system. Together these changes are reflected physiologically as a primarily supralaryngeal response via narrowing of the supraglottic area in response to the perturbed laryngeal airway resistance. These four subjects may be profiled as a similar group characterized by a strong resonance strategy with a generally passive respiratory strategy in response to perturbation.

Subjects 02, and 06 also demonstrated more passive response from the respiratory systems; however these two subjects had very small respiratory excursions in all conditions indicating a very small range of respiratory volume was used habitually by these subjects for voicing even in the PRE condition. There were very small changes in respiratory strategy between conditions for these subjects. Pressure and flow changed minimally between conditions $(0.18 \mathrm{~cm} \mathrm{H} 20$ and $30 \mathrm{~mL} / \mathrm{S}$ for subject 002 , and 0.92 $\mathrm{cmH} 20$ and $-10 \mathrm{~mL} / \mathrm{s}$ for subject 06 ). There were also only small changes in formant spacing for these subjects. These two subjects demonstrated a combined respiratory and resonance strategy; however there was not a large change in laryngeal airway resistance for these two subjects. These subjects represent a second profile characterized by a small but combined respiratory and resonance strategy.

Subjects 03, 04, 05, and 07 generally had larger changes in airway resistance than the other subjects and demonstrated varied, but generally more active responses from the respiratory system.

Subjects 03, 04, and 07 had respiratory initiations and terminations above EEL before perturbation. Subject 05 had abdominal terminations below EEL in the PRE 
condition indicating active abdominal contribution under their normal voicing circumstances.

Subject 03 had lower RC and $A B$ initiations and terminations that dropped below EEL in the PAR condition. There were small changes in the supralaryngeal component. Overall this subject had a predominantly respiratory strategy in response to the decrease in airway resistance.

Subject 04 had lower $R C$ and $A B$ initiations with the $A B$ initiation dropping below EEL. Both RC and $A B$ terminations were well below EEL indicating a very strong respiratory response to perturbation. F5 - F4 decreased by 56\% and F2 - F1 increased by $192 \%$. This participant increased $\mathrm{P}_{\mathrm{s}}$ by $1.77 \mathrm{cmH} 20$ and flow by $400 \mathrm{~mL} / \mathrm{s}$. There was a strong respiratory and supralaryngeal response to this large decrease to LAR.

Subject 05 had a $1.14 \mathrm{cmH} 20$ increase in $\mathrm{P}_{\mathrm{s}}$ and an $80 \mathrm{~mL} / \mathrm{s}$ increase in flow. This subject had an increase in spacing in the upper formants and a large increase in the space between F2 - F1. AB initiation and termination decreased indicating a more active abdominal strategy. Ribcage terminations also were below EEL indicating that both $\mathrm{RC}$ and $\mathrm{AB}$ musculature was more active in order to maintain pressure in response to increased airflow. This subject had a predominant respiratory strategy to cope with increased airflow with a small increase in supralaryngeal activity.

Subject 07 had a $1.88 \mathrm{cmH} 20$ decrease in $\mathrm{P}_{\mathrm{s}}$ and a $30 \mathrm{~mL} / \mathrm{s}$ increase in flow. None of the formant changes were greater than $10 \%$ between conditions. This subject had a substantial change in respiratory strategy in the PAR condition. In the PRE condition there was a greater $\mathrm{RC}$ contribution where both $\mathrm{RC}$ and $\mathrm{AB}$ initiations and terminations were well above EEL. During the PAR condition there was a greater AB 
contribution. Both $R C$ and $A B$ initiations were lower, but above EEL. AB terminations were below EEL indicating an active abdominal response.

Overall, these subjects demonstrated varied but strong respiratory responses to perturbation. Although each individual demonstrated a unique response to perturbation, general similarities among subjects indicated a strong combined supralaryngeal and respiratory response, a strong supralaryngeal response, or a strong respiratory response. These individuals represented a third profile because of their strong and varied respiratory responses. Because this group had larger changes (with the exception of subject 07) in airflow during perturbation, the compensatory strategies in this group were larger.

Visual representations of the individual changes to the three subsystems are presented in Figures 5.1 - 5.5. These tables plot the percent change scores between the PRE and PAR conditions by subject for the three subsystems of phonation and highlight individual differences between the two conditions. Figure 5.6+ displays kinematic motion plots of individual changes in ribcage and abdomen contributions across the PRE, PAR and REC conditions. These plots provide greater detail of the respiratory strategies among the three conditions by individual. 
Figure 5.1 Percent Changes by Subject for Formant Spacing Between PRE and PAR Conditions.

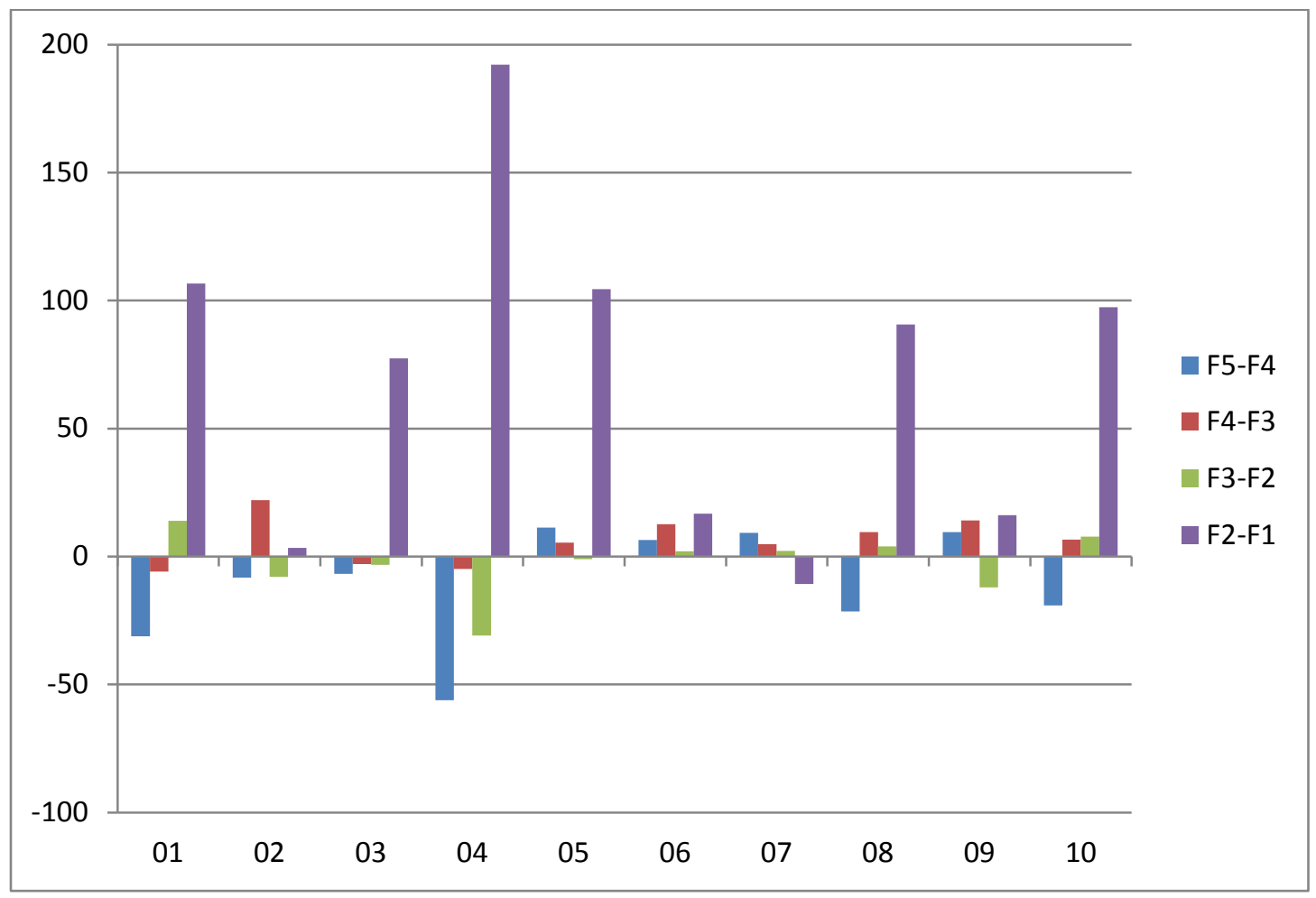


Figure 5.2

Percent Changes by Subject for Aerodynamic Measures from PRE to PAR ( $P_{s}$, Flow).

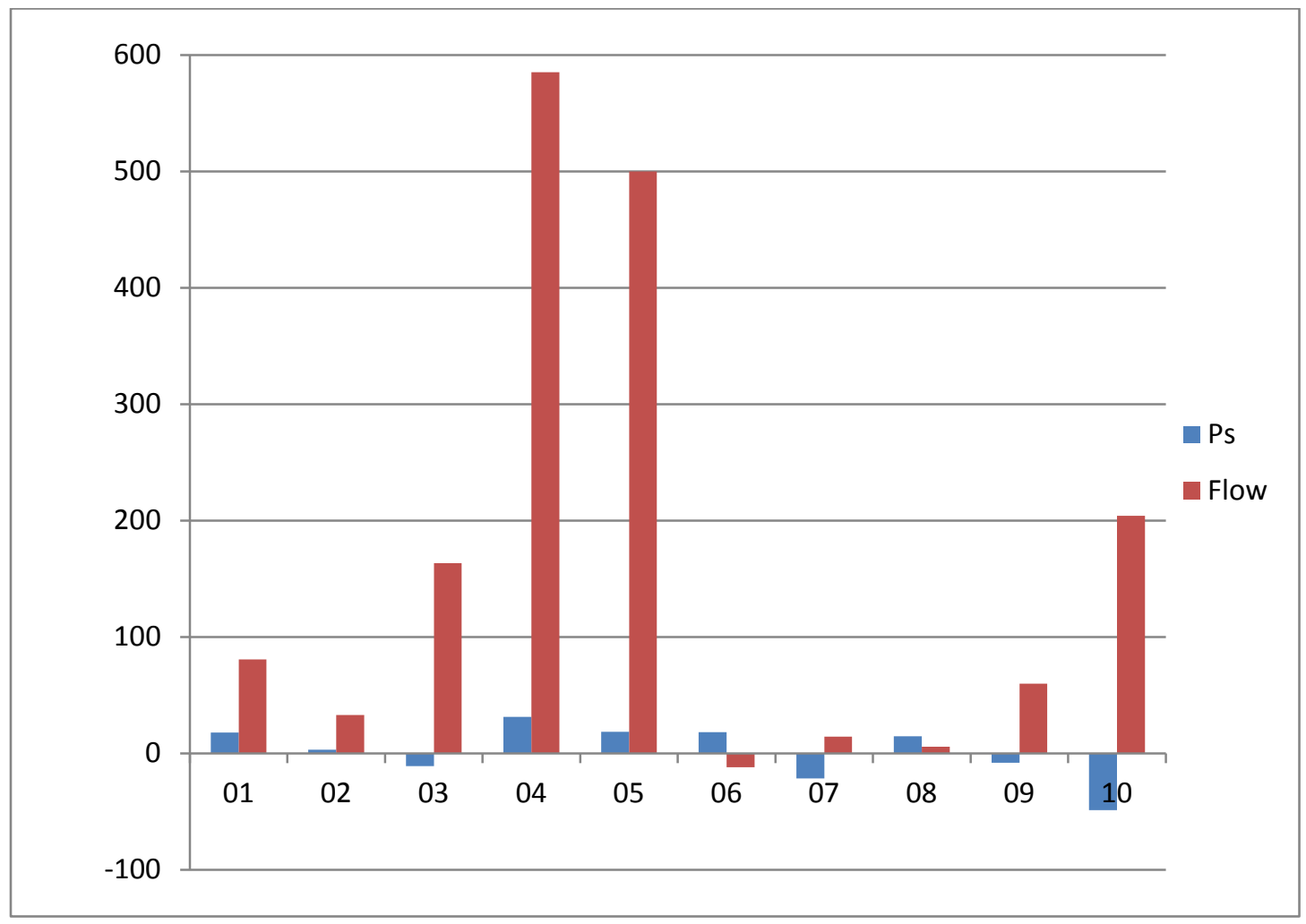

Figure 5.3

Percent Change by Subject from PRE to PAR for Lung Volume Initiation, Termination, and Excursion (LVI, LVT, LVE)

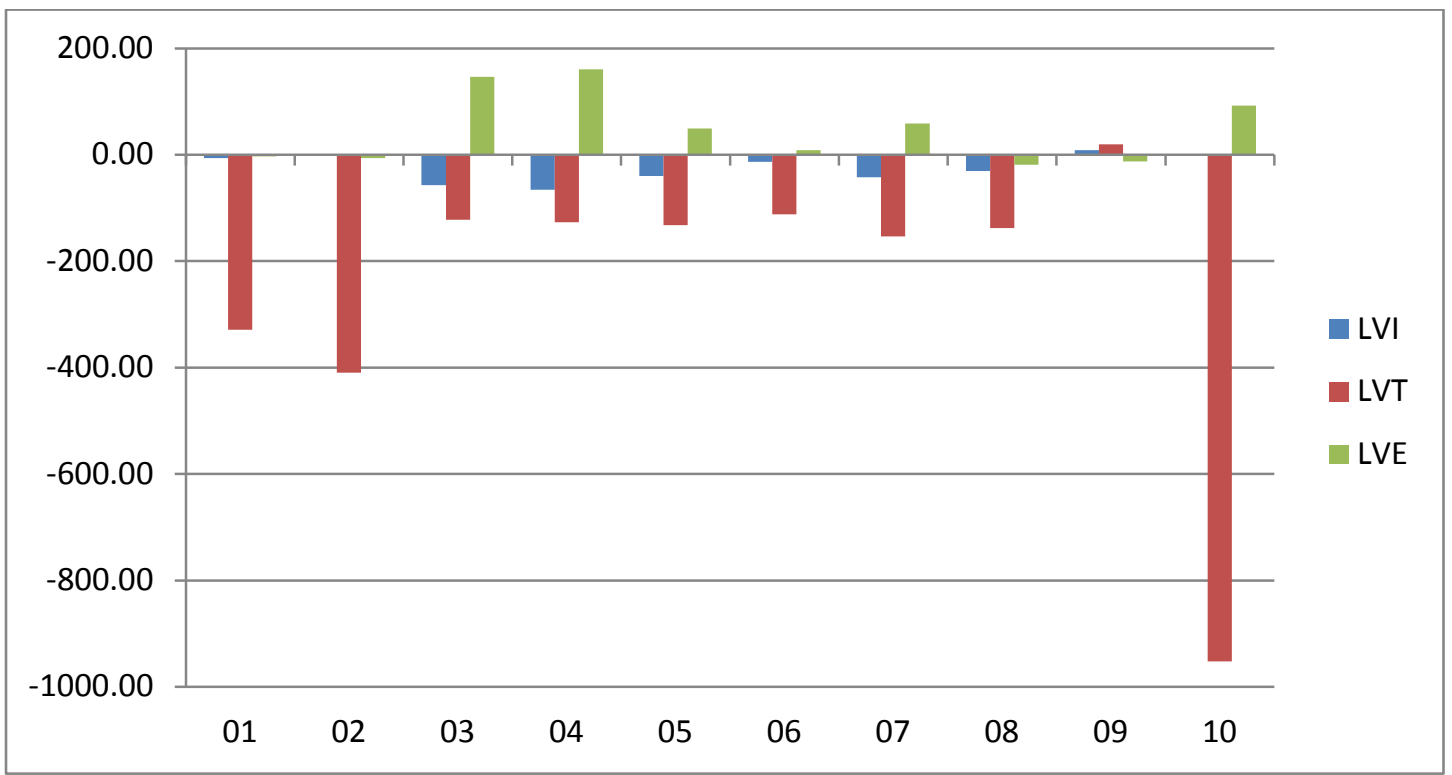


Figure 5.4

Percent Change by Subject from PRE to PAR for Rib Cage Initiation, Termination, and Excursion (RCl, RCT, RCE)

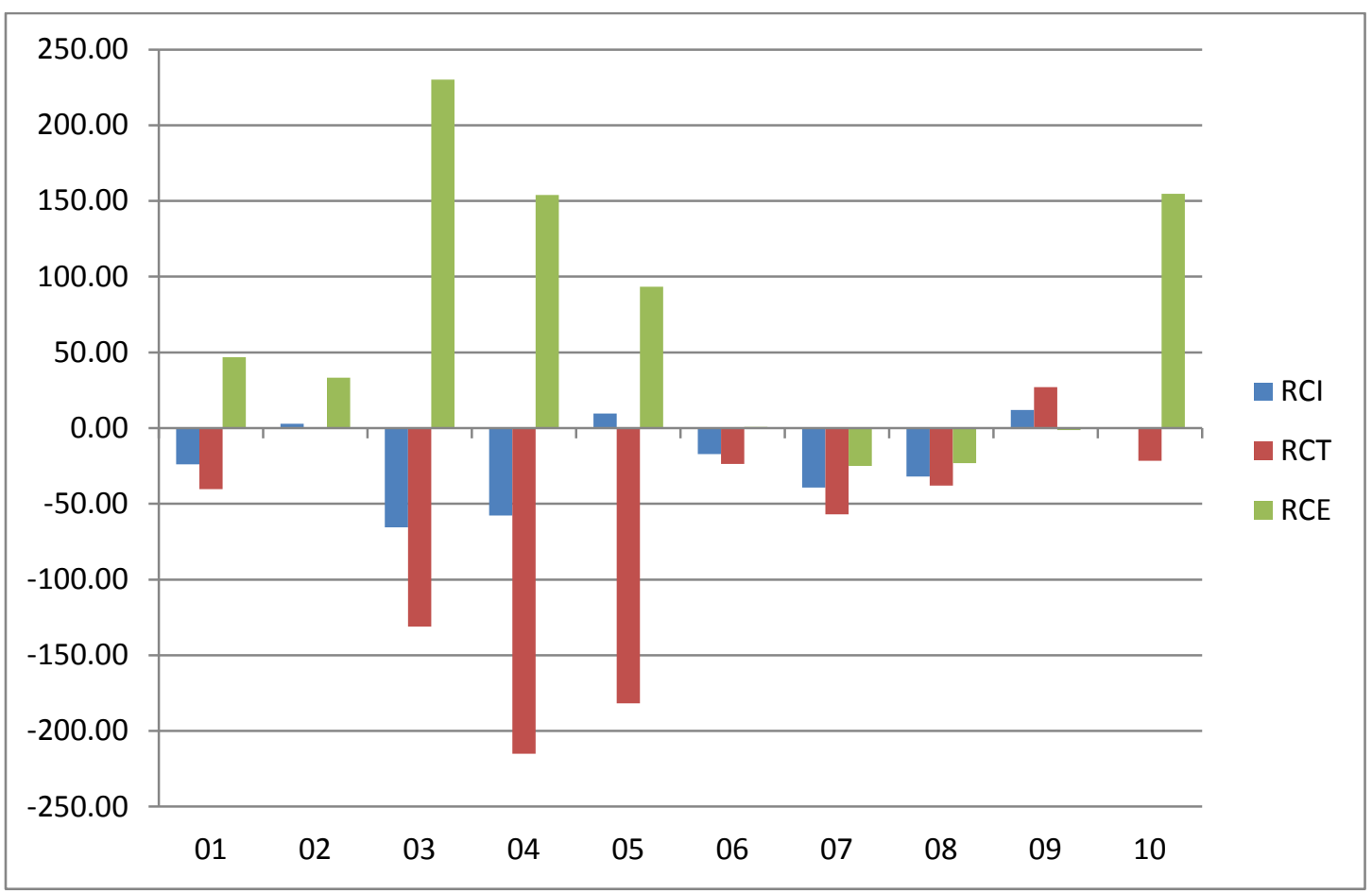

Figure 5.5 Percent Change for PRE to PAR by Subject for Abdominal Initiation, Termination, and Excursion ( $\mathrm{ABI}, \mathrm{ABT}, \mathrm{ABE})$

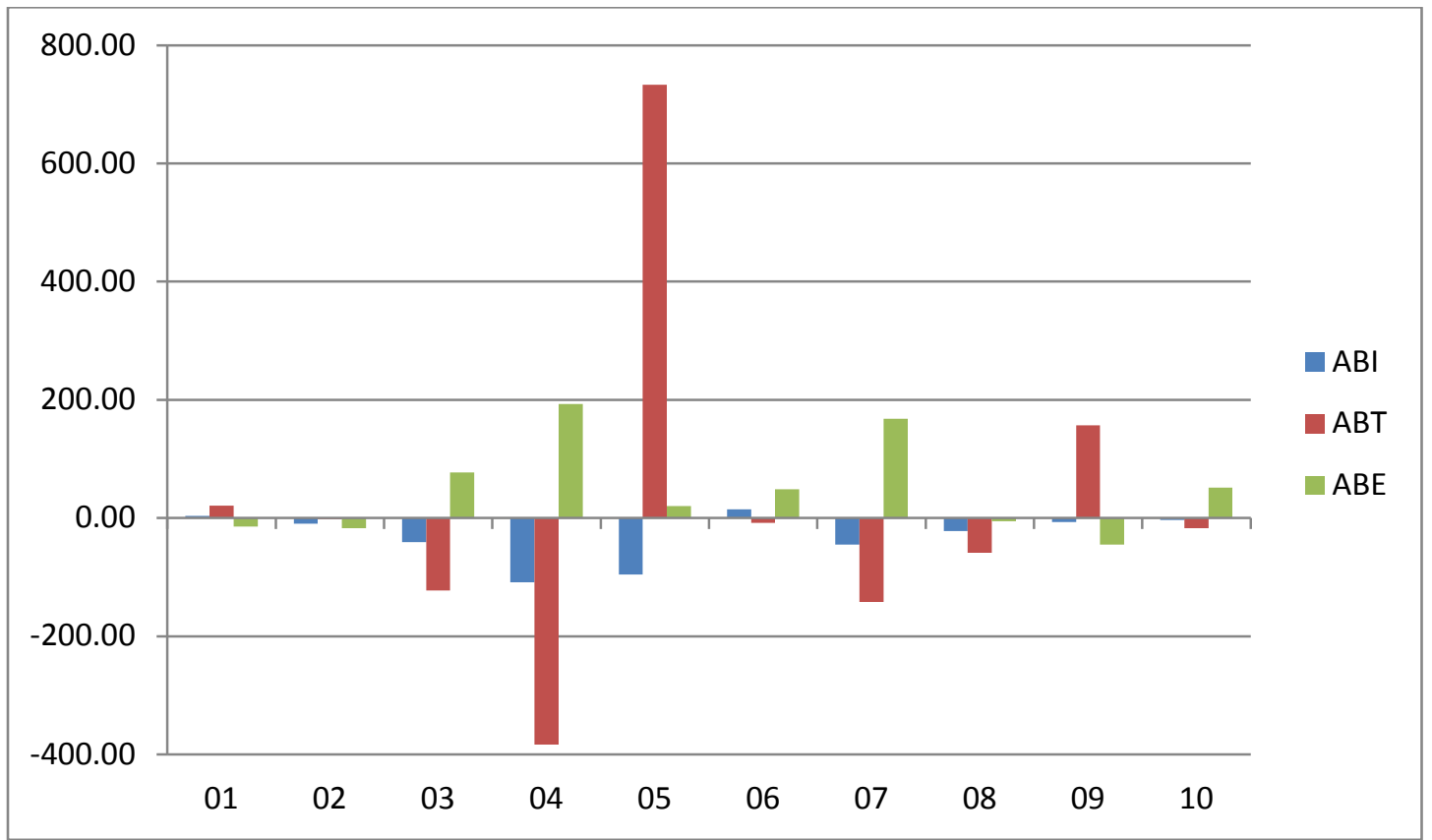




\section{Figure 5.6+}

Respiratory Kinematic Plots by Subject: Rib cage volume (y-axis) by abdominal volume (x-axis) average motion $\mathrm{x}$ motion for each participant during the three voicing conditions.

Blue $=$ PRE, Red $=$ DUR, and Green $=$ REC. Upper right symbols indicate mean utterance initiation. Lower left symbols indicate mean utterance termination. EEL for rib cage and abdomen is represented by 0 at cross-axis.
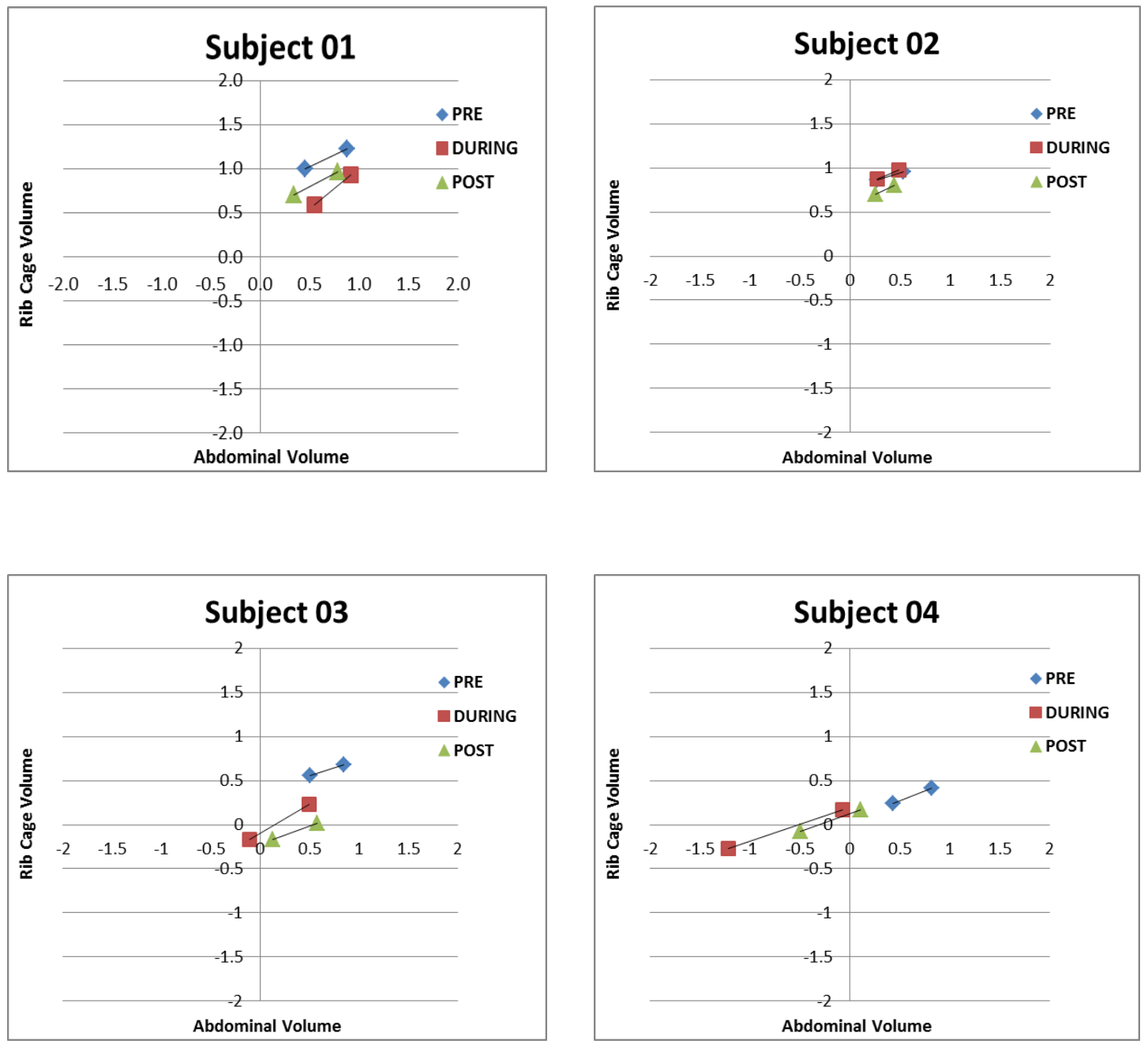

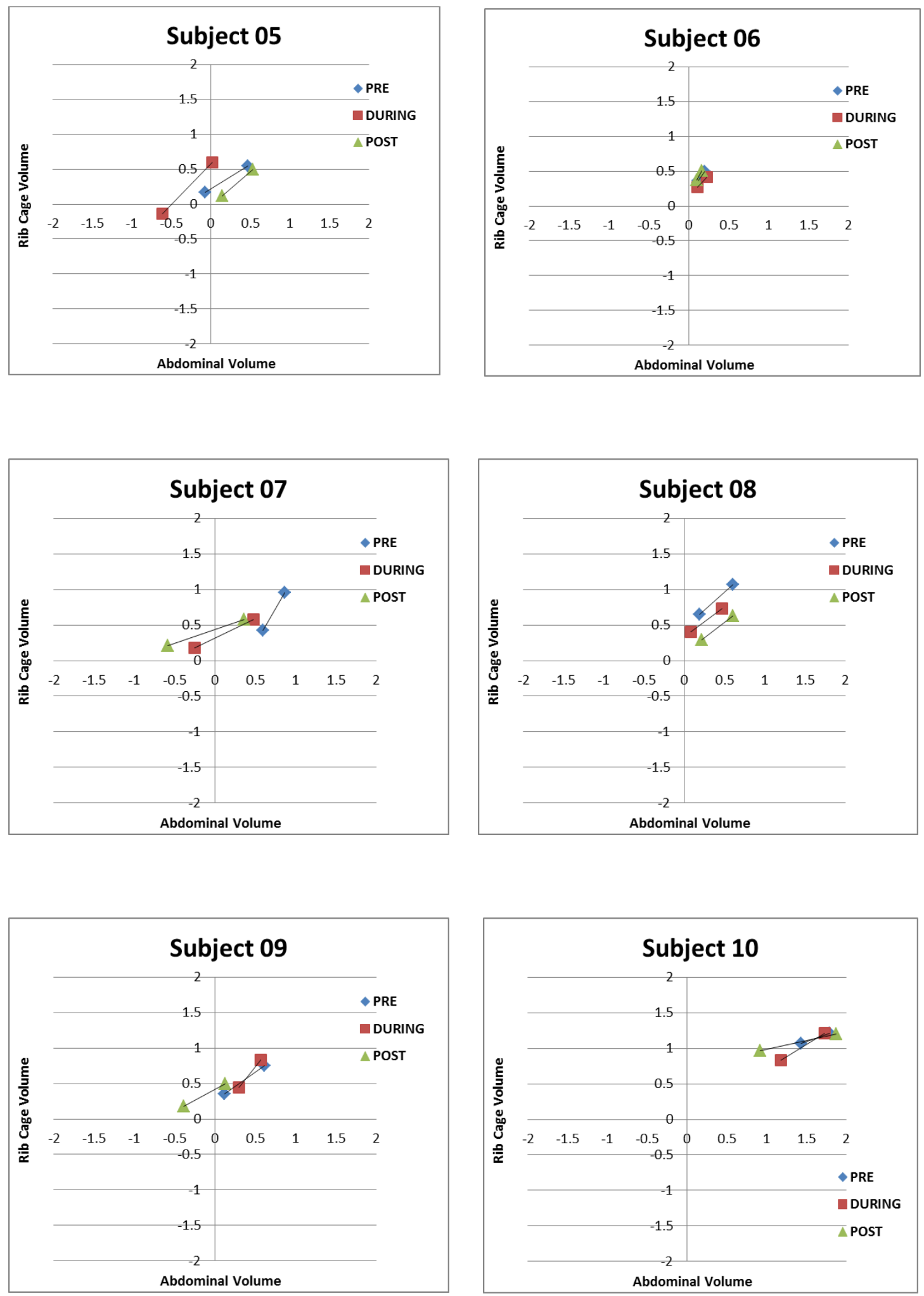


\section{Summary}

The interactive nature of the vocalization subsystems observed in this study could not be observed by interpretation of the individual subsystems alone.

Simultaneous and representative measures of all subsystems were necessary to view and interpret the relationships among the subsystems both within and across individuals. Additionally, group averages hid important details necessary to capture the differences among individuals. Some important observations from this study include:

1. Resonance changes where characterized by a narrowing of the spacing of the upper formants and a widening of the spacing of the lower formants. This is physiologically significant from what is known about the effects of epilaryngeal narrowing.

Physiologically, some degree of supraglottic constriction in the epilarynx area was noted for all subjects in the PAR condition.

2. Despite similar positions of the paralytic vocal fold (paramedian in all cases) very different alterations in airflow rate resulted.

3. Subglottic pressure was generally maintained across all conditions indicating that a primary compensatory strategy in response to glottic incompetence is maintenance of subglottic pressure from the resulting increase airflow rates.

4. Respiratory strategy varied greatly by subject. A more varied and active respiratory response was generally observed with greater decrease in laryngeal airway resistance. All but one participant had lower lung volume initiations indicating that taking advantage of passive recoil of the respiratory system was not a preferred response to decreased laryngeal resistance. 
5. Ribcage and abdominal proportional contributions varied markedly in response to increased airflow rate becoming more variable with greater airflow change.

6. Not all subjects returned to their baseline subsystem vocalization strategy in the recovery phase. This may be due to the fact that measures were taken only a few minutes after confirming returned vocal fold mobility. Another possibility for this is that the paralytic state may function as a quite stable attractor pattern. This will be discussed more below in relation to dynamic systems theory. A Glossary of relevant terms regarding DST is located in Table 5.1. 
Table 5.1 Terms relating to discussion of Dynamic Systems Theory

\begin{tabular}{ll}
\hline Term & Definition \\
\hline Collective Variable(s) & Variables that are used to describe an \\
& emergent phenomenon which are selected \\
& as a representative compression of the \\
& many variables which could express system \\
& behavior. These variables are selected to \\
& describe/measure the contextual emergence \\
& of a behavior and represent only a few of \\
& the many potential variables acting on a \\
& system. Stated differently, these variables \\
& represent a resultant behavior of interest \\
& and are chosen from many possibilities to \\
& observe/measure that behavior. A collective \\
& variable expresses an underlying pattern of \\
& interest in a complex behavior. \\
& Attractor states are preferred patterns of \\
behavior in a complex system which result \\
from the cooperation of the many elements \\
of a system in a given context. These are \\
stable modes of operation which are not \\
programmed, but rather emergent given the \\
context and constraints of a system. These \\
states can shift to another preferred mode if \\
the context or constraints of the system are \\
altered. This is termed a phase shift. \\
Control parameters govern the internal \\
cohesion of a dynamic system. A control \\
parameter may be an element or condition, \\
but is of critical importance to the stability of \\
system function. These parameters have \\
critical limits, which if crossed will disrupt the \\
systems integrity and cause a shift in \\
performance.
\end{tabular}

\section{Dynamic Systems Theory Applied}

We have discussed the differences and similarities in the group data and by subject. Earlier it was discussed that viewing the subsystems of phonation as separated boxes with a bottom-up trajectory is not an adequate model of phonation because it does not well represent the interactive nature of the subsystems. A more appropriate and dynamic model should demonstrate the proportional and interactive nature of the contributions among the subsystems. Using group data from a representative measure from each subsystem, a new conceptual model based in dynamic systems was created. 
Figures 5.7 - 5.9 are three-dimensional graphs plotting the spatial relationships from the most influential variables from each subsystem according to the standardized data. These variables most actively contributed to change across conditions (Respiration = RCT, Phonation $=$ Flow, and Resonance $=$ F2 - F1). Graphs from the PRE, DUR, and REC conditions plot the normalized data for each participant from each subsystem in a three-dimensional state space. The state space is a theoretical three-dimensional plot which represents all of the observations of a variable's value plotted as a trajectory in space. One representative variable from each subsystem was plotted to visually represent its own trajectory in the state space and also its relationship to the other variables in space. The average data point for each subject, for each chosen representative measure was plotted on a corresponding plane creating a cloud of data on each plane. These clouds in the state space are the attractor basins for each voicing condition. Previously, we discussed attractors as stable states in which a system prefers to function in a given context. Ellipses were drawn around the data points to aid in visualization of the subsystem attractors and their trajectory vector in the state space, and also to represent the degree of overlap among attractor patterns for the subsystem triad.

Figure 5.7 plots the collective variables in the state space for the three representative variables in the PRE condition. The individual points tend to cluster together for each subsystem variable with their trajectories forming a small amount of central overlap, but generally are orthogonal to each other. This may be considered a representation of "balanced" phonation where no one subsystem particularly dominates the state space and represents a stable mode of function.

Figure 5.8 plots the same representative variables in the DUR condition. The variables tend to overlap considerably more centrally, and the trajectory vectors as 
demonstrated by the direction of the ellipses around the data points for each subsystem remained similar. During the paralytic condition the changes in the variables caused greater interaction among the variables represented by the central overlap of the collective variables in the state space.

Figure $\mathbf{5 . 9}$ plots the variables in the REC phase. Here, there remained significant overlap in the subsystems, however the individual data points demonstrated less organization within each subsystem and are more scattered across the state space. The vector trajectories of the collective variables has changed as evidences by the direction of the ellipses surrounding the data points.

These state space plots demonstrate some interesting relationships among the variables in terms of attractor patterns (stable modes of system operation). First, in the PRE condition the individual subsystems demonstrate a clear grouping of data points with little overlap among subsystems, which may indicate a stable attractor pattern which is characteristic of normal voicing.

In the PAR condition, the trajectories of the variables in each subsystem is maintained; however the interaction among the systems becomes much more integrated, as indicated by the degree of overlap among the subsystems. This indicates a different yet very stable attractor pattern that characterizes the PAR condition.

In the REC condition, the individual data points in each subsystem become less organized as noted by a lack of clustering and changing trajectories of the data points within the subsystems; however the overlap among the systems is still very evident. This may indicate an unstable transitional state as most subjects did not completely return to their PRE state data values, especially in terms of respiratory kinematics. 
In terms of dynamic systems, a point of interest is that the audible dysphonia (hoarseness) that was characteristic of the paralytic state appears to be a very strong attractor pattern as evidenced by the similar trajectories of the variables between conditions, but with much greater overlap in their interactions. Additionally, most subjects did not return to baseline function after the PAR condition. This further provides evidence that dysphonia is a strong attractor state, possibly even more influential than the "normal" voice state. Why would this be?

One possibility in favor of the idea of a stable dysphonic attractor state is often observed clinically, as patients that have developed acute dysphonia secondary to upper respiratory infection often have difficulty returning to their normal voice quality after the infection has cleared. Similarly, patients that receive injection laryngoplasty for glottic incompetence (to close the glottic valve) also often require additional voice therapy after the injection because they hold onto dysphonic patterns of phonation. Moreover, it has been demonstrated that even perceptually normal voices can function even more efficiently after voice training/therapy. It may be that "normal" voice is not the most stable attractor state and is easily pushed into a new mode of operation with even mild perturbation.

One way to potentially explain the paradox that "normal" may not be the strongest attractor state of voice production is through the concepts of vocal efficiency vs. vocal economy ${ }^{73}$. A "pressed" voice (one with high laryngeal resistance at the glottis generally and associated with muscular strain) is quite efficient in terms of vocal output power from a strictly mechanical standpoint. Stated differently, this configuration produces a louder voice with carrying power. This would make a voice that is "pressed" or produced with a high degree of laryngeal resistance a very strong attractor. However, this kind of vocal production is not very economical to the voice user because it comes 
at the cost of tissue injury of the vocal fold mucosa, resulting in vocal fatigue, hoarseness, and potential pathological lesions. A voice that is economical in a human being would benefit from maximizing vocal output power while minimizing vocal strain and effort. What is interesting is that this ideal balance often does not happen without voice training. The biomechanical target of voice therapy is to maximize vocal output with less vocal effort. ${ }^{74}$ If this occurred naturally and without training, economical voice production would not need to be cultivated. This means that the stronger attractor in voice production may be a voice that is more mechanically efficient, but also one that is not necessarily economical to the voice user.

\section{Lessons in Vocal Control in Relation to Dynamic Systems}

As stated earlier, Dynamic Systems Theory does not seek to give priority to any one component of a system as a deterministic regulator of system function. Instead it is the cooperative interplay of the elements that result in an emergent behavior. ${ }^{13,75,76}$ However, when elements combine they typical do not display all the theoretically existent possibilities, but rather tend to "collect" into stable modes of operation on observation. A collective variable, or variables are ones that capture a complex phenomenon by reducing many degrees of freedom into a few that are representative of the behavior. In our case the three representative variables chosen represent and capture the interaction of the three subsystems and create a "landscape" in space that demonstrates vocal output under differing conditions. Using this, we can see when the system changes (in this case by discrete perturbations).

In order to determine how the system changes, it is important to identify potential control parameter variables/conditions that capture how the system responds to changes in the collective variable landscape. We chose to perturb the system by disrupting the 
laryngeal valve during voicing, which resulted in increased airflow rates due to air leak through the glottis. It appears that an important control parameter that helps regulate the vocal system is maintenance of subglottic pressure. This was evidenced by the maintenance of both subglottic pressure and sound pressure level at the lips in response to decreased resistance at the glottis from the increased airflow. Neither of these variables were significantly different during the PAR condition. In order to maintain subglottic (and vocal tract acoustic pressure) alterations in respiratory kinematics and supraglottic configuration were observed. It appears that $P_{s}$ then, is an important control parameter which results in alterations of both the respiratory and resonance systems in order to actively maintain $\mathrm{P}_{\mathrm{s}}$. Airflow rate (although necessary for pressure maintenance) does not seem as critical to overall system function given that flow rates in both normal and disordered voices result in pressure adjustments that maintain a relatively consistent subglottic pressure. Additionally, in the normal voice, wide variability exists in the range of "normal" airflow rates $(60-200+\mathrm{mL} / \mathrm{s})$ and despite this wide range, subglottic pressure is generally maintained. 
Figures 5.7 - 5.9. Scatterplots with resonance on the x-axis (green), respiration on the $y$-axis (blue), and phonation on the z-axis (red). Ellipses have been constructed around the data points to help demonstrate the spatial relationships among the three subsystems in each voicing condition. Graphs have been rotated to maximize view of the overlap among the systems.

Figure 5.7

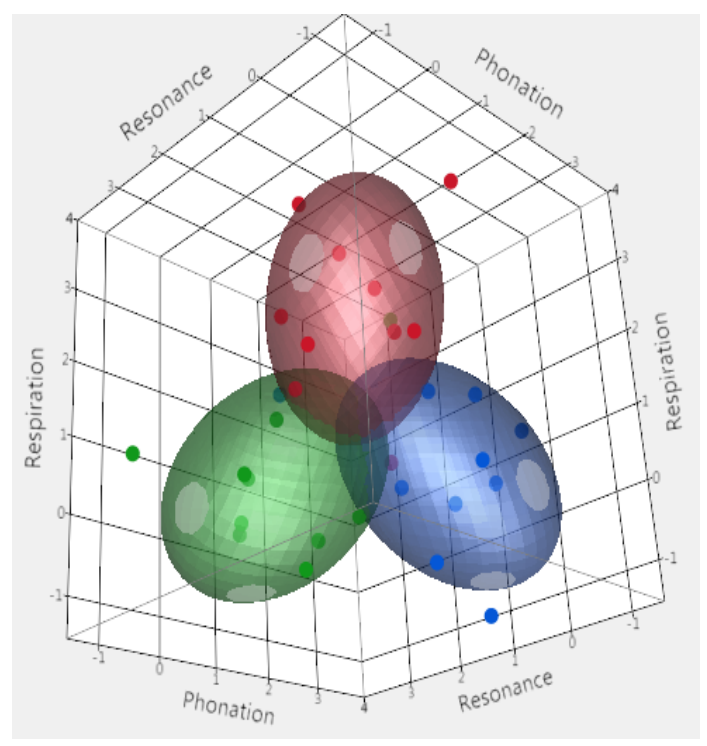

Figure 5.9

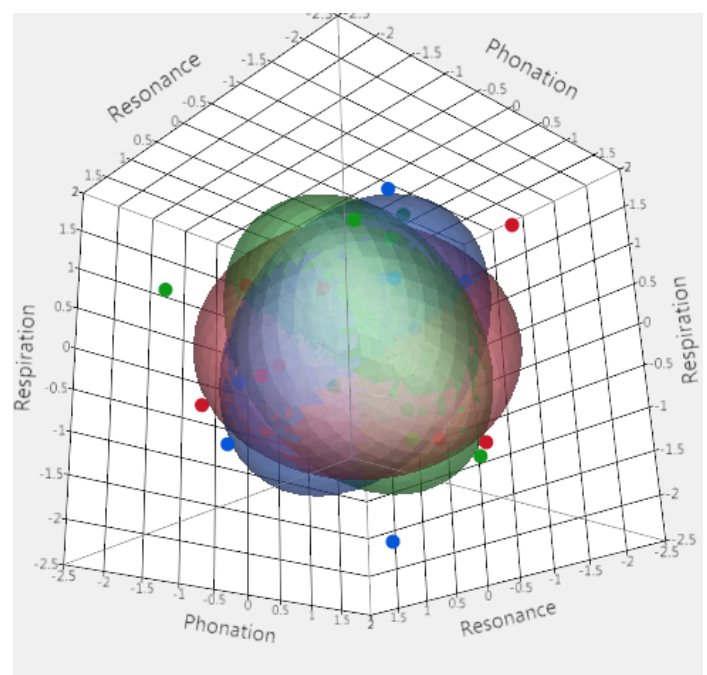

Figure 5.8

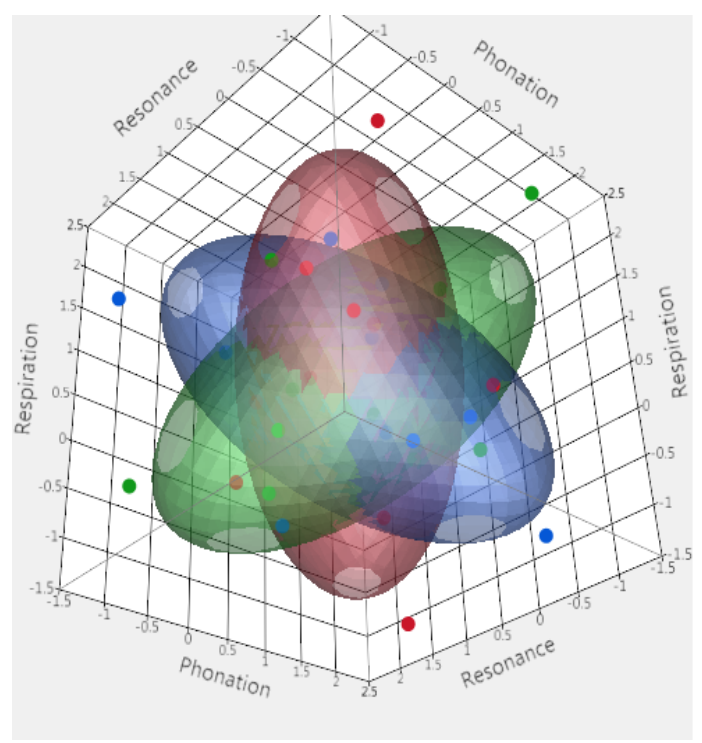




\section{Clinical Implications}

Clinically, the individual differences noted in this study are important in order to translate into clinical practice. It is clear that individuals compensate differently in response to laryngeal perturbation and understanding the interactive nature of the three subsystems can help direct personalized intervention strategy. It is important to consider that practically, both normal and pathological voice conditions exist on a continuum. Data from this study are immediately translational, as therapeutic techniques already exist to treat dysfunction of the subsystems. Information from this study can better direct treatment strategy as an improved holistic picture can be drawn from the individual data.

This study highlights the need for a revision of the three box model of phonation. A model which better depicts the interactive changes among the three subsystems is warranted given the increasing understanding of the process of vocalization. More studies characterizing both normal and disordered voicing will help determine a more appropriate model which demonstrates how individual variables shape the overall contour of the model. A more appropriate model may be depicted by a Venn diagram where overlap and size of the components could represent the predominant strategy of an individual.

\section{Application to Personalized Medicine}

The individual differences in performance observed during this study were deemed important because of the longstanding idea that individuals likely regulate the phonatory subsystems differently during vocalization, and these differences could be of clinical significance. This study permitted qualitative and quantitative evidence that individuals do indeed have differing regulatory strategies in both normal and disordered states. These findings indicate that identifying differences and commonalities in clinical 
patients is important in order to provide timely, efficient, and effective treatment to patients with voice disorders.

In medicine, personalized treatment has become a topic of intense interest as evidence is mounting that patient's do not benefit from a "one size fits all" treatment approach. Averages are not always appropriate when judging effectiveness of a drug for a specific treatment across individuals. For example, diabetes drugs have been shown to be ineffective in $43 \%$ of an average sample. Even more staggering, is that cancer drugs are ineffective in $75 \%$ of cases on average. ${ }^{77}$ Because of the notion that averages do not represent the population well in terms of treatment, personalized medicine attempts to tease out the individual differences in human biology to provide more effective treatments for the individual. Applied to voice disorders, personalized treatment is envisioned to encompass a variety of issues including:

- Directing selection of appropriate and optimal treatment strategies from an already available arsenal of tools, and the opportunity to develop new improved treatments.

- Increasing patient adherence and decreasing dropout rate by providing more rapid and effective results.

- $\quad$ Providing preventative tools to those who are at occupational risk for a voice disorder such as teachers.

- Improvement in quality of life.

- Reducing costs of treatment by reducing the number of treatment visits.

- Improved indication to payors, providers, and patients that the therapy provided is necessary and efficacious for a particular patient. 
The future of personalized treatment for voice disorders will necessitate a revised model of voice production which takes individual differences into account. It will also require more studies with larger subject populations that observe the entire vocalization system with analyses of both average and individual data. This type of multi-measure methodology has the potential to bring our field closer to the reality of disorder specific treatments for a variety of voice disorders. The hope of this type of assessment and treatment approach is to move beyond treating the disorder and effectively treating the person.

While promising, personalized voice treatment does have practical challenges because the amount of testing required to make decisions based on a particular individual. The advantage in the field of voice disorders when compared to other fields, is that effective assessment and treatment techniques are already available. With the exception of respiratory kinematic measures, multi-dimensional voice assessment is feasible in many clinics, as cost-effective and high quality instrumentation is now available and measures can be acquired and interpreted quickly by the treating clinician. Additionally, physiologic voice therapy (treatment of the underlying physiologic impairment in a voice disorder) has been demonstrated to be effective for treatment of many vocal pathologies; however increasing knowledge of the causal determinants of voice disorders has the potential to further refine current therapeutic techniques.

\section{Limitations}

A primary limitation of this study is lack of statistical power due to small sample size. The decision of a small sample was a tradeoff so that the sample would remain small enough to make sense of individual differences observed in the study. Our sample size limits inferential interpretation and generalizability. This study is also limited by a 
lack of male subjects. Differences in sex have been described in the literature; however these determinations could not be made within our sample. Future studies should include a more equal male to female ratio for comparisons. Findings of this study are in accordance with established theories of voice production, but should be interpreted with caution until larger studies and replication of these results can confirm our findings.

\section{Delimitations}

The decision not to control for frequency and intensity in order to provide experimental control during this study was made in order to intentionally observe how subjects would respond to a perturbation without constraints placed by the investigator. It was felt that this was a more realistic and externally valid representation of normal and disordered voice production.

\section{Future Directions}

In order to revise the current model of voice production, future studies should consider use of similar measurement methodology in a large number of normal subjects in order map the boundaries of normal phonation and determine the individual differences which occur during normal voice production. This simultaneous multimeasure methodology should also be used to describe different pathological conditions leading to disorder specific treatments. Additionally, in order to further enhance treatment strategies this same methodology could be used to study patients both preand post-treatment in order to determine how our current therapy is changing individual physiology over the course of treatment. 


\section{APPENDICIES}

The following appendices provide results of correlational analyses and detailed analyses of the individual data in this study. 


\section{APPENDIX 1}

\section{Correlations for change scores \\ PRE to PAR conditions}

Because mean airflow rate was found to significantly increase in all subjects, it was of interest to determine if there were correlations with increased airflow and the other dependent variables. Correlations were calculated on PRE/PAR and PAR/REC group data to determine how the variables changed with increasing/decreasing airflow in the paralytic condition and during the recovery condition. Physiologic correlates to these changes are discussed in chapter 5.

\section{Acoustic measures}

1. F5 - F4: There was a significant correlation between F5-F4 and flow. As flow increased, the space between F5-F4 decreased. $\left[p=0.0052\left(r^{2}=0.64\right)\right]$.

2. F4-F3: There was a non-significant correlation between F4-F3 and flow demonstrating that as flow increased the space between F4 and F3 decreased, $\left[p=0.1951\left(r^{2}=0.199\right)\right]$.

3. F3-F2: There was a non-significant correlation between F3-F2 and flow indicating that as flow increased the space between F3 and F2 decreased. [ $\left.p=0.0792\left(r^{2}=0.335\right)\right]$.

4. F2- F1: There was a significant correlation between F2-F1 and flow indicating that as flow increased the space between F2 and F1 increased. $\left[p=0.0174\left(r^{2}=0.53\right)\right]$.

\section{Aerodynamic measures}

1. Subglottic pressure: There was not a significant relationship or trend between pressure and flow between conditions. This was expected as pressure did not significantly change across conditions. $\left[p=0.83\left(r^{2}=0.006\right)\right]$. 


\section{Respiratory measures}

1. LVI: There was not a significant relationship or trend between LVI and flow between conditions. [p = $\left.0.95\left(r^{2}=0.0004\right)\right]$.

2. LVT: There was a non-significant trend between LVT and flow. As flow increased LVT decreased. $\left[p=0.53\left(r^{2}=0.052\right)\right]$.

3. LVE: LVE tended to increase with increased flow; however this was not significant. [p $\left.=0.088\left(r^{2}=0.32\right)\right]$.

4. $R C l$ : There was not a significant relationship or trend between $\mathrm{RCl}$ and flow. $[\mathrm{p}=0.79$ $\left.\left(r^{2}=0.009\right)\right]$.

5. RCT: There was not a significant relationship between RCT and flow. There was a trend that demonstrated that as flow increased, RCT decreased. [ $\left.p=0.19\left(r^{2}=0.21\right)\right]$.

6. RCE: There was a significant correlation between RCE and flow. As flow increased RCE also increased. [ $\left.p=0.023\left(r^{2}=0.49\right)\right]$.

7. $A B I$ : There was a significant correlation between $A B I$ and flow indicating that as flow increased ABI decreased. $\left[p=0.046\left(r^{2}=0.41\right)\right]$.

8. $A B T$ : There was a significant correlation between $A B T$ and flow. As flow increased ABT decreased. $\left[p=0.014\left(r^{2}=0.55\right)\right]$.

9. ABE: There was a significant correlation between $A B E$ and flow. As flow increased ABE also increased. $\left[p=0.008\left(r^{2}=0.61\right)\right]$. 


\section{PAR to REC conditions}

1. F5-F4: There was a non-significant correlation between the space between F5 and F4 with flow. As flow decreased the space between F5 and F4 Increased. [p $=0.32$ $\left(r^{2}=0.12\right)$.

2. F4- F3: There was no significant correlation between F4 - F3 and flow. A slight trend indicated that as flow decreased formant space increased. [p $\left.=0.36\left(r^{2}=0.10\right)\right]$.

3. F3-F2: There was not a significant correlation between F3-F2 and flow. There was a trend indicating that as flow decreased the space between f3 and f2 also decreased. [p = $\left.0.27\left(r^{2}=0.15\right)\right]$.

4. F2-F1: there was not a significant correlation between F2-F1 and flow. There was a trend indicating that as flow decreased the space between F2 and F1 increased. [p = $\left.0.40\left(r^{2}=0.09\right)\right]$.

1. Subglottic Pressure: There was not a significant correlation or trend between pressure and flow. $\left[p=0.59\left(r^{2}=0.04\right)\right]$.

1. LVI: There was not a significant correlation or trend between LVI and flow. [0.80 $\left.\left(r^{2}=0.009\right)\right]$.

2. LVT: There was not a significant correlation or trend across LVT and flow. [0.85 $\left(r^{2}=\right.$ 0.005)]

3. LVE: There was a non-significant trend between flow and LVT. Generally as flow decreased LVE decreased. [ $\left.p=0.38\left(r^{2}=0.09\right)\right]$.

4. $R C l$ : There was not a significant correlation or trend between $\mathrm{RCl}$ and flow. $[\mathrm{p}=0.91$ $\left(r^{2}=0.002\right)$. 
5. RCT: There was a non-significant correlation between RCT and Flow. Generally as flow decreased RCT was more positive. $\left[p=0.50\left(r^{2}=0.06\right)\right]$.

6. RCE: There was a significant correlation between flow and RCE. Generally as flow decreased RCE also decreased. [p = $\left.0.0098\left(r^{2}=0.59\right)\right]$.

7. $A B I$ : There was a non-significant correlation between $A B I$ and flow. Generally as flow decreased, $A B I$ increased. $\left[p=0.34\left(r^{2}=0.11\right)\right]$.

8. $A B T$ : There was a non-significant correlation between $A B T$ and Flow. Generally as flow decreased ABT became more positive. $\left[p=0.28\left(r^{2}=0.14\right)\right]$.

9. ABE: There was a non-significant correlation between RCE and flow. As flow decreased ABE generally decreased. 


\section{APPENDIX 2}

\section{RESULTS BY SUBJECT: Individual Subject Profiles}

\section{PRE - PAR Conditions}

Subject 01

Acoustic Measures

Subject 01, demonstrated the greatest changes in F5-F4 (-31\%) and F2-F1 (106\%) and demonstrated an overall trend for a narrowing of the spacing of formants above $\mathrm{F} 2$ and an increase in spacing between F1 and F2.

\section{Aerodynamic Measures}

Aerodynamic measures indicated a small increase in pressure $[1.41 \mathrm{~cm} / \mathrm{H} 20$ (18\%)] and an increase in flow [100 mL/s (81\%)].

\section{Respiratory Kinematic Measures}

Respiratory measures indicated LVI decreased slightly (-6\%), LVT was more negative (-32\%), and LVE decreased slightly across conditions (-3\%). The contribution of the abdomen was generally predominant in the PAR condition. Kinematic plots revealed a decrease in $\mathrm{RCI}(-24 \%), \mathrm{RCT}(-40 \%)$ and an increase in RCE (47\%). There was a slight increase in $A B I(4 \%), A B T(21 \%)$ and a shorter $A B E(-15 \%)$. This participant used a mostly passive respiratory response of both the rib cage and abdomen (both remained in the upper right quadrant of the kinematic plot) in response to increased airflow in order to maintain subglottic pressure despite a lower LVI and $\mathrm{RCl}$ during perturbation. 
Subject 02

Acoustic Measures

Subject 02 demonstrated a decrease in F5-F4 (-8\%) and in F3-F2 (-8\%) and a very small increase in the spacing between F1 and F2 (3.4\%).

\section{Aerodynamic Measures}

Subglottic pressure increased marginally (3\%). Airflow increased by $80 \%$.

\section{Respiratory Kinematic Measures}

Subject 02 used very little respiratory support as evidenced by small excursions. The predominant breathing pattern was a greater abdominal contribution overall. Changes in respiratory strategy during the PAR condition were a small decrease in LVI (-2\%), large decrease in LVT (-410\%), and a shorter LVE (-6\%). RCI and RCT both increased marginally ( $3 \%$ and $0.6 \%$ respectively). RCE increased by $33 \%$. ABI, ABT and AVE were all decreased $(-10 \%,-2 \%$, and $-17 \%$ respectively).

Subject 03

\section{Acoustic Measures}

Subject 03 demonstrated narrowing of upper formant spacing. [F5-F4 (-7\%), F4F3 (-3\%), and F3-F2 (-3\%)] There was a large increase in the space between F1 and F2 $(77 \%)$.

\section{Aerodynamic Measures}

Aerodynamic measures indicated a $1 \mathrm{cmH} 20$ (-11\%) drop in pressure and 160 $\mathrm{mL} / \mathrm{s}$ increase in flow (163\%). 


\section{Respiratory Kinematic Measures}

Subject 03 had lower much lower LVI, and LVT (-57\%, and -121\%) and increased LVE (147\%). RCI and RCT were lower (-66\%, and -130\%) with increased RCE (230\%). ABI and ABT were decreased (-41\%, and -123\%) and ABE was increased (77\%).

Both ribcage and abdominal terminations were below EEL during perturbation indicating active action from the muscles of forced expiration. Kinematic plots indicated a general predominance of abdominal strategy over ribcage for this participant.

\section{Subject 04}

\section{Acoustic Measures}

Subject 04 demonstrated a decrease in upper formant spacing [(F5-F4, -56\%; F4-F3, -5\%; and F3-F2, -31\%)] and a large increase in spacing between F1 and F2 (192\%).

\section{Aerodynamic Measures}

Aerodynamic changes demonstrated a $1.77 \mathrm{cmH} 20$ (31\%) increase in pressure and a substantial $400 \mathrm{~mL} / \mathrm{S}(585 \%)$ increase in airflow.

\section{Respiratory Measures}

Subject 04 changed respiratory strategy markedly between conditions. LVI and LVT decreased markedly (-66\% and $-127 \%)$. LVE was increased by $160 \%$. RCI and RCT were decreased (-58\% and -215\%). RCE was increased by $153 \%$. ABI and ABT were decreased (-109\% and -384\%). ABE increased by $192 \%$. This subject used their abdominal muscles to compensate for increased airflow as $\mathrm{ABI}$ and $\mathrm{ABT}$ fell well below 
EEL indicating active abdominal contraction to maintain pressure. RCT was also below EEL indicating active muscle contraction. This subject had a strong respiratory and laryngeal response to maintain pressure in response to increased airflow during perturbation.

Subject 05

\section{Acoustic Measures}

Acoustic data demonstrated that the spacing between upper formants increased by a small amount ( $15 \%$ overall) and that the space between F1 and F2 increased markedly (104\%).

\section{Aerodynamic Measures}

This subject increased subglottic pressure by $1.14 \mathrm{~cm} \mathrm{H} 20$ (18\%) and demonstrated a $500 \%(80 \mathrm{~mL} / \mathrm{s})$ increase in airflow during the PAR condition.

\section{Respiratory Kinematic Measures}

For this subject, LVI and LVT were decreased (-40\% and -133\%) with LVE increased by $49 \%$. RCI increased slightly by $10 \%$, and RCT decreased markedly ($182 \%)$. RCE increased by $93 \%$. ABI was decreased (-96\%) while ABT was largely increased (732\%). ABE was increased by $20 \%$. RCI shifted from well above EEL to near EEL in the PAR condition. This subject had ABTs below EEL in the PRE condition and increased this strategy to well below EEL in the PAR condition. Both RCT and ABT were well below EEL in the PAR condition indicating active expiratory muscle effort. Kinematic plots indicated a change from a greater abdominal contribution to greater ribcage predominance from PRE to PAR conditions. 
Subject 06

Acoustic Measures

There was a small overall increase in upper formants $(-22 \%)$ and a small increase in the spacing between F1 and F2 (17\%).

\section{Aerodynamic Measures}

Aerodynamic measures indicated a small increase in pressure $[(0.92 \mathrm{cmH} 20$, $(18 \%)]$ and a small decrease in flow $[(10 \mathrm{~mL} / \mathrm{s},(-12 \%)]$. This was the only subject whose flow rate decreased in the PAR condition.

\section{Respiratory Kinematic Measures}

LVI and LVT were decreased (-13\% and -112\%) and LVE was increased by $9 \%$. $\mathrm{RCl}$ and $\mathrm{RCT}$ were decreased (-17\% and $-24 \%)$. RCE was essentially maintained with a less than $1 \%$ increase. $A B I$ was increased (15\%), while $A B T$ was decreased by $-8 \%$. ABE was increased by $49 \%$. Kinematic plots indicated very small excursions in all conditions. Initiations and terminations for all variables were minimally above EEL, and the very small kinematic excursions indicate a weak respiratory drive. Kinematic plots indicated a greater ribcage contribution to the respiratory strategy used by this subject.

\section{Subject 07}

\section{Acoustic Measures}

There was a small increase in the upper formants ( 16\%) and a small decrease in F2-F1 (-11\%). 


\section{Aerodynamic Measures}

Aerodynamic data indicated a $1.88 \mathrm{cmH} 20(-22 \%)$ decrease in pressure and a small increase in average flow by $30 \mathrm{~mL} / \mathrm{s}(14 \%)$.

\section{Respiratory Kinematic Measures}

Respiratory strategy changed markedly between conditions for this subject. LVI and LVT decreased (-42\%, and -153\%) LVE was increased by 59\%. RCI, RCT and RCE all decreased (39\%, 57\%, and $-25 \%$ respectively). ABI and $A B T$ were decreased $(-45 \%$, and $-142 \%)$. ABE was increased markedly by $168 \%$. Kinematic plots indicated a marked change in respiratory strategy between conditions. Plots also indicated a shift from a greater ribcage contribution to a larger abdominal contribution. Ribcage terminations remained above EEL while abdominal volumes terminated below EEL indicating active activation of the abdominal muscles in the PAR condition.

\section{Subject 08}

\section{Acoustic Measures}

Acoustic measures indicated a decrease the space between F5-F4 (-22\%) There was a mild increase in spacing between F2 and F4 ( 14\%). There was a large increase in the spacing between F1 and F2 (91\%).

\section{Aerodynamic Measures}

Aerodynamic measures indicated a small increase in pressure $[1.3 \mathrm{cmH} 20$, (15\%)]. Airflow increased by $6 \%$. 


\section{Respiratory Kinematic Measures}

Respiratory measures indicated a decrease in all lung volume measures LVI, LVT, and LVE decreased by $-30 \%,-137 \%$, and $-19 \%$ respectively. $\mathrm{RCI}, \mathrm{RCT}$, and RCE decreased by $-32 \%,-37 \%$, and $-23 \%$. ABI, ABT, and ABE all decreased by $-22 \%,-58 \%$, and $-5 \%$. Kinematic plots indicated all initiations and terminations to be above EEL indicating a passive respiratory strategy with a slight increase in abdominal contribution in the PAR condition.

Subject 09

\section{Acoustic Measures}

There was a small (10\%) increase in F5-F4, a small increase in F4-F3 (14\%), and a decrease in F3-F2 (-12\%). There was a small increase in the space between F1 and F2 (16\%).

\section{Aerodynamic Measures}

Aerodynamic measures demonstrated a small decrease in pressure [-0.38 cmH20. (-8\%)], and a $40 \mathrm{~mL} / \mathrm{s}(60 \%)$ increase in airflow.

\section{Respiratory Kinematic Measures}

LVI, and LVT increased (8\% and 19\%) while LVE decreased by $-13 \% . \mathrm{RCI}$ and RCT increased (12\%, and 27\%). RCE decreased minimally by $-1 \%$. ABI decreased by $7 \%$. ABT increased by $157 \%$, and ABE decreased by $-45 \%$. This participant used

primarily passive recoil the of ribcage with active activation of the abdominal muscles to maintain pressure in the PAR condition. 
Subject 10

Acoustic Data

Acoustic data indicated a decrease in F5-F4 (-19\%) and small increase in F4-F3, and F3-F2 (7\% and 8\%), and a large increase in the space between F1 and F2 (97\%).

\section{Aerodynamic Measures}

Aerodynamic data revealed a $3.63 \mathrm{cmH} 20$ (49\%) decrease in pressure and a 160 $\mathrm{mL} / \mathrm{s}(204 \%)$ increase in airflow.

\section{Respiratory Kinematic Measures}

LVI, and LVT decreased (-1\% and 151\%), LVE increased by $92 \%$. RCI was not significantly changed (0.1\%). RCT was decreased (-21\%), and RCE was increased by 154\%. $\mathrm{ABI}$ and $\mathrm{ABT}$ were decreased (-4 and $-18 \%)$ and $\mathrm{ABE}$ was increased by $51 \%$. There was a predominant abdominal strategy in all conditions. Kinematic plot data indicated a generally passive strategy as all measures were above EEL. 


\section{References}

1. Mangravite LM, Thorn CF, Krauss RM. Clinical implications of pharmacogenomics of statin treatment. The pharmacogenomics journal. 2006;6(6):360-374.

2. Rieder MJ, Reiner AP, Gage BF, et al. Effect of VKORC1 haplotypes on transcriptional regulation and warfarin dose. The New England journal of medicine. 2005;352(22):2285-2293.

3. Spear BB, Heath-Chiozzi M, Huff J. Clinical application of pharmacogenetics. Trends in molecular medicine. 2001;7(5):201-204.

4. Terra SG, Hamilton KK, Pauly DF, et al. Beta1-adrenergic receptor polymorphisms and left ventricular remodeling changes in response to betablocker therapy. Pharmacogenetics and genomics. 2005;15(4):227-234.

5. Shah SH, Arnett D, Houser SR, et al. Opportunities for the Cardiovascular Community in the Precision Medicine Initiative. Circulation. 2016;133(2):226-231.

6. Isshiki N. Regulatory mechanism of voice intensity variation. Journal of speech and hearing research. 1964;7:17-29.

7. Kent RD. The speech sciences. San Diego, CA: Singular; 1997.

8. Stemple J, Glaze L, Klaben B. Clinical voice pathology: Theory and management. 3rd ed. San Diego: Singualr Publishing group; 2000.

9. Netsell, Lotz W, DuChane A, Barlow S. Vocal tract aerodynamics during syllable productions: Normative data and theoretical implications. Journal of Voice. 1991;5(1):1-9.

10. Zraick RI, Smith-Olinde L, Shotts LL. Adult normative data for the KayPENTAX Phonatory Aerodynamic System Model 6600. J Voice. 2012;26(2):164-176.

11. Hixon TJ. Kinematics of the chest wall during speech production: volume displacements of the rib cage, abdomen, and lung. Journal of speech and hearing research. 1973;16(1):78-115.

12. Peterson G, Barney $\mathrm{H}$. Control methods used in a study of the vowels. Journal of the Acoustical Scoiety of America. 1952;24:175-184.

13. Thelen E, Ulrich BD. Hidden skills: a dynamic systems analysis of treadmill stepping during the first year. Monographs of the Society for Research in Child Development. 1991;56(1):1-98; discussion 99-104.

14. Glasgow RE, Magid DJ, Beck A, Ritzwoller D, Estabrooks PA. Practical clinical trials for translating research to practice: design and measurement recommendations. Medical care. 2005;43(6):551-557.

15. Tunis SR, Stryer DB, Clancy CM. Practical clinical trials: increasing the value of clinical research for decision making in clinical and health policy. JAMA : the journal of the American Medical Association. 2003;290(12):1624-1632. 
16. Lee, Stemple JC, Kizer M. Consistency of acoustic and aerodynamic measures of voice production over 28 days under various testing conditions. $J$ Voice. 1999;13(4):477-483.

17. Fang FC, Casadevall A. Reductionistic and holistic science. Infection and immunity. 2011;79(4):1401-1404.

18. Fardet A, Rock E. The search for a new paradigm to study micronutrient and phytochemical bioavailability: from reductionism to holism. Medical hypotheses. 2014;82(2):181-186.

19. Gatherer D. So what do we really mean when we say that systems biology is holistic? BMC systems biology. 2010;4:22.

20. Mazzocchi F. Complexity and the reductionism-holism debate in systems biology. Wiley interdisciplinary reviews. Systems biology and medicine. 2012;4(5):413427.

21. Hixon TJ, Mead J, Goldman MD. Dynamics of the chest wall during speech production: function of the thorax, rib cage, diaphragm, and abdomen. Journal of speech and hearing research. 1976;19(2):297-356.

22. Chadha TS, Watson $\mathrm{H}$, Birch S, et al. Validation of respiratory inductive plethysmography using different calibration procedures. The American review of respiratory disease. 1982;125(6):644-649.

23. Sackner MA, Watson $\mathrm{H}$, Belsito AS, et al. Calibration of respiratory inductive plethysmograph during natural breathing. J Appl Physiol (1985). 1989;66(1):410420.

24. Hoit JD, Hixon TJ. Body type and speech breathing. Journal of speech and hearing research. 1986;29(3):313-324.

25. Hoit JD, Hixon TJ. Age and speech breathing. Journal of speech and hearing research. 1987;30(3):351-366.

26. Winkworth AL, Davis PJ, Adams RD, Ellis E. Breathing patterns during spontaneous speech. Journal of speech and hearing research. 1995;38(1):124144.

27. Winkworth AL, Davis PJ, Ellis E, Adams RD. Variability and consistency in speech breathing during reading: lung volumes, speech intensity, and linguistic factors. Journal of speech and hearing research. 1994;37(3):535-556.

28. Rahn $\mathrm{H}$, Otis $A B$, Chadwick LE, Fenn WO. The pressure-volume diagram of the thorax and lung. American Journal of Physiology. 1946;146:161-178.

29. Russell NK, Stathopoulos E. Lung volume changes in children and adults during speech production. Journal of speech and hearing research. 1988;31(2):146-155.

30. Hodge MM, Rochet AP. Characteristics of speech breathing in young women. Journal of speech and hearing research. 1989;32:466-480. 
31. D. HJ, Hixon TJ, Altman ME, Morgan WJ. Speech breathing in women. Journal of speech and hearing research. 1989;32:353-365.

32. Wilder $\mathrm{CN}$. Chest wall preparation for phonation in female speakers. In: Bless $\mathrm{DM}$, Abbs JH, eds. Vocal fold physiology-contemporary research and clinical issues. San Diego: College-Hill Press; 1983:109-123.

33. Stathopoulos ET, Sapienza CM. Respiratory and laryngeal function of men and women during vocal intensity variation. Journal of speech and hearing research. 1993;36:64-75.

34. Stathopoulos ET, Sapienza CM. Respiratory and laryngeal measures of children during vocal intensity variation. Journal of the Acoustical Society of America. 1993;94:2531-2543.

35. Huber JE, Stathopoulos ET. Respiratory and laryngeal responses to an oral air pressure bleed during speech. J Speech Lang Hear Res. 2003;46(5):1207-1220.

36. Smitheran JR, Hixon TJ. A clinical method for estimating laryngeal airway resistance during vowel production. The Journal of speech and hearing disorders. 1981;46(2):138-146.

37. Rothenberg M. A new inverse-filtering technique for deriving the glottal air flow waveform during voicing. The Journal of the Acoustical Society of America. 1973;53(6):1632-1645.

38. Collyer S, Davis PJ. Effect of facemask use on respiratory patterns of women in speech and singing. J Speech Lang Hear Res. 2006;49(2):412-423.

39. Huber JE, Stathopoulos ET, Bormann LA, Johnson K. Effects of a circumferentially vented mask on breathing patterns of women as measured by respiratory kinematic techniques. J Speech Lang Hear Res. 1998;41(3):472-478.

40. Hirano M. Clinical examination of voice. New York: Springer; 1981.

41. Tanaka S, Gould WJ. Relationships between vocal intensity and noninvasively obtained aerodynamic parameters in normal subjects. The Journal of the Acoustical Society of America. 1983;73(4):1316-1321.

42. Awan SN, Novaleski CK, Yingling JR. Test-retest reliability for aerodynamic measures of voice. J Voice. 2013;27(6):674-684.

43. Baken RJ, Orlikoff RF. Clinical Measurement of Speech and Voice. 2nd ed. San Diego, CA: Singular Publishing Group; 2000.

44. Miller JD. Auditory-perceptual interpretation of the vowel. The Journal of the Acoustical Society of America. 1989;85(5):2114-2134.

45. Sundberg J. Articulatory interpretation of the singing formant. Journal of the Acoustical Society of America. 1974;55:838-844.

46. Bennett S. Vowel formant frequency characteristics of preadolescent males and females. The Journal of the Acoustical Society of America. 1981;69(1):231-238. 
47. Fant G. Acoustic theory of speech production. Netherlands: Mouton \& Co. N.V.; 1960.

48. Sulter AM, Wit HP. Glottal volume velocity waveform characteristics in subjects with and without vocal training, related to gender, sound intensity, fundamental frequency, and age. The Journal of the Acoustical Society of America. 1996;100(5):3360-3373.

49. Titze I. Nonlinear source-filter interaction in singing. Physiology of Acoustics and Singing Conference; 2004; Denver. CO.

50. Titze I, Riede T, Popolo P. Nonlinear source-filter coupling in phonation: vocal exercises. The Journal of the Acoustical Society of America. 2008;123(4):19021915.

51. Titze IR. Voice training and therapy with a semi-occluded vocal tract: rationale and scientific underpinnings. J Speech Lang Hear Res. 2006;49(2):448-459.

52. Guzman M, Laukkanen AM, Krupa P, Horacek J, Svec JG, Geneid A. Vocal tract and glottal function during and after vocal exercising with resonance tube and straw. J Voice. 2013;27(4):523 e519-534.

53. Harrison P. Variability of Formant Measurements. New York: Language and Linguistic Science, University of New York; 2004.

54. Titze IR. Nonlinear source-filter coupling in phonation: theory. The Journal of the Acoustical Society of America. 2008;123(5):2733-2749.

55. Sapienza CM, Stathopoulos ET. Speech task effects on acoustic and aerodynamic measures of women with vocal nodules. J Voice. 1995;9(4):413418.

56. Stathopoulos ET, Sapienza CM. Developmental changes in laryngeal and respiratory function with variations in sound pressure level. J Speech Lang Hear Res. 1997;40(3):595-614.

57. Dromey C, Ramig LO, Johnson AB. Phonatory and articulatory changes associated with increased vocal intensity in Parkinson disease: a case study. Journal of speech and hearing research. 1995;38(4):751-764.

58. Stathopoulos ET, Hoit JD, Hixon TJ, Watson PJ, Solomon NP. Respiratory and laryngeal function during whispering. Journal of speech and hearing research. 1991;34(4):761-767.

59. Stathopoulos ET, Huber JE, Richardson K, et al. Increased vocal intensity due to the Lombard effect in speakers with Parkinson's disease: simultaneous laryngeal and respiratory strategies. Journal of communication disorders. 2014;48:1-17.

60. Gillespie Al, Gartner-Schmidt J, Rubinstein EN, Verdolini Abbott K. Aerodynamic Profiles of Females with Muscle Tension Dysphonia/Aphonia. J Speech Lang Hear Res. 2012.

61. Dedo HH. Recurrent laryngeal nerve section for spastic dysphonia. The Annals of otology, rhinology, and laryngology. 1976;85(4 Pt 1):451-459. 
62. Izdebski K, Shipp T, Dedo HH. Predicting postoperative voice characteristics of spastic dysphonia patients. Otolaryngology and head and neck surgery. 1979;87(4):428-434.

63. Ludlow CL, Naunton RF, Bassich CJ. Procedures for the selection of spastic dysphonia patients for recurrent laryngeal nerve section. Otolaryngology--head and neck surgery : official journal of American Academy of Otolaryngology-Head and Neck Surgery. 1984;92(1):24-31.

64. Roy N, Smith ME, Allen B, Merrill RM. Adductor spasmodic dysphonia versus muscle tension dysphonia: examining the diagnostic value of recurrent laryngeal nerve lidocaine block. The Annals of otology, rhinology, and laryngology. 2007;116(3):161-168.

65. Smith ME, Roy N, Wilson C. Lidocaine block of the recurrent laryngeal nerve in adductor spasmodic dysphonia: a multidimensional assessment. Laryngoscope. 2006;116(4):591-595.

66. Joshi A, Jiang Y, Stemple JC, Archer SM, Andreatta RD. Induced unilateral vocal fold paralysis and recovery rapidly modulate brain areas related to phonatory behavior: a case study. J Voice. 2011;25(2):e53-59.

67. Holmberg EB, Hillman RE, Perkell JS. Glottal airflow and transglottal air pressure measurements for male and female speakers in soft, normal, and loud voice. The Journal of the Acoustical Society of America. 1988;84(2):511-529.

68. Behroozmand R, Korzyukov O, Sattler L, Larson CR. Opposing and following vocal responses to pitch-shifted auditory feedback: evidence for different mechanisms of voice pitch control. The Journal of the Acoustical Society of America. 2012;132(4):2468-2477.

69. Shrout PE, Fleiss JL. Intraclass correlations: uses in assessing rater reliability. Psychological bulletin. 1979;86(2):420.

70. Rosner B. Fundamental of biostatistics. Belmont, CA: Duxbury Press; 2005.

71. Sundberg J, La FM, Gill BP. Formant tuning strategies in professional male opera singers. J Voice. 2013;27(3):278-288.

72. Sapienza CM, Stathopoulos ET, Brown WS, Jr. Speech breathing during reading in women with vocal nodules. J Voice. 1997;11(2):195-201.

73. Titze I. Quantifying vocal efficiency and economy - how can computation augment clinical assessment? Proceedings of Meetings on Acoustics. 2013;19(1):060244.

74. Verdolini K, Druker DG, Palmer PM, Samawi H. Laryngeal adduction in resonant voice. J Voice. 1998;12(3):315-327.

75. Thelen E. Development as a dynamic system. Current Directions in Psychological Science. 1992;1(6):189-193.

76. Thelen E, Smith LB. A dynamic systems approach to the development of cognition and action. MIT press; 1996. 
77. Abrahams E, Silver M. The case for personalized medicine. Journal of diabetes science and technology. 2009;3(4):680-684. 


\section{VITA}

Daniel J. Croake

\section{Date and Place of Birth}

Date of birth: November 3, 1977

Place of birth: Middletown, Ohio

\section{Education}

Doctoral candidate, Rehabilitation Sciences, successfully defended dissertation on $11 / 09 / 2016$

December, 2016

University of Kentucky

Dissertation: Vocalization Subsystem Responses to a Temporarily Induced Vocal Fold

Paralysis

Cognate in Clinical and Translational Science

May 2014

University of Kentucky

Master's in Speech Language Pathology

May 2010

University of Kentucky

Thesis: The consistency of the LEMG signal in normal subjects

Master's in Music

May 2004

Ohio University

\section{Professional Positions}

Speech-Language Pathologist

University of Kentucky Voice and Swallow Clinic

January 2015 - Present

Graduate Assistant to Joseph C. Stemple,

Dept. of Communication Sciences and Disorders, University of Kentucky

January, 2011 - July, 2011

Clinical Doctoral Fellowship

Central Baptist Hospital

August, $2010-2014$

\section{Scholastic and professional honors}

Invited Speaker, Kentucky Speech-Language and Hearing Association (2015)

Undergraduate Research Enhancement Award (2014)

Graduate School Travel Award, University of Kentucky (2011)

Research Assistant, University of Kentucky Clinical Voice Center (2009) 


\section{Professional Publications}

1. Croake, D., Andreatta, R., \& Stemple, J. (2016, August). Immediate effects of the vocal function exercises semi-occluded mouth posture on glottal airflow parameters: A preliminary study. Journal of Voice.

2. Angadi, V., Croake, D., \& Stemple, J. (2016, In Review). Efficacy of vocal function exercises: a systematic review. Journal of Voice.

3. Croake, D., Stemple, J., Uhl, T., Archer, S., \& Andreatta, R. (2014, April). Reliability of clinical office-based laryngeal electromyography in vocally healthy adults. Annals of Otology, Rhinology, and Laryngology. 